\title{
Bat Diversity in Cat Ba Biosphere Reserve, Northeastern Vietnam: A Review with New Records from Mangrove Ecosystem
}

\author{
Vu Dinh Thong ${ }^{1,2, *}$, Annette Denzinger ${ }^{3}$, Nguyen Van Sang ${ }^{4}$, Nguyen Thi Thu Huyen ${ }^{4} \mathbb{C}_{\text {, Hoang Trung Thanh }}{ }^{4}$, \\ Dao Nhan Loi ${ }^{5}$, Pham Van Nha ${ }^{5}$, Nguyen Van Viet ${ }^{6}$, Pham Duc Tien ${ }^{1}$, Mao-Ning Tuanmu ${ }^{7}{ }^{1}$, \\ Joe Chun-Chia Huang ${ }^{7} \mathbb{D}$, Ladthavong Thongphachanh ${ }^{5}$, Nguyen Thanh Luong ${ }^{1}$ and Hans-Ulrich Schnitzler ${ }^{3}$
}

\section{check for} updates

Citation: Thong, V.D.; Denzinger, A. Sang, N.V.; Huyen, N.T.T.; Thanh, H.T.; Loi, D.N.; Nha, P.V.; Viet, N.V.; Tien, P.D.; Tuanmu, M.-N.; et al. Bat Diversity in Cat Ba Biosphere Reserve, Northeastern Vietnam: A Review with New Records from Mangrove Ecosystem. Diversity 2021, 13, 376 https://doi.org/10.3390/d13080376

Academic Editors: Christoph Meyer, Sergei V. Kruskop and Luc Legal

Received: 15 July 2021

Accepted: 7 August 2021

Published: 14 August 2021

Publisher's Note: MDPI stays neutral with regard to jurisdictional claims in published maps and institutional affiliations.

Copyright: (C) 2021 by the authors Licensee MDPI, Basel, Switzerland This article is an open access article distributed under the terms and conditions of the Creative Commons Attribution (CC BY) license (https:// creativecommons.org/licenses/by/ $4.0 /)$
1 Institute of Ecology and Biological Resources, Vietnam Academy of Science and Technology (VAST), 18 Hoang Quoc Viet Road, Cau Giay District, Hanoi 10072, Vietnam; tienphd@gmail.com (P.D.T.); t19012@gmail.com (N.T.L.)

2 Faculty of Ecology and Biological Resources, Graduate University of Science and Technology, Vietnam Academy of Science and Technology (VAST), 18 Hoang Quoc Viet Road, Cau Giay District, Hanoi 10072, Vietnam

3 Animal Physiology, Institute for Neurobiology, Faculty of Sciences, University of Tübingen, Auf der Morgenstelle 28, D-72076 Tübingen, Germany; annette.denzinger@uni-tuebingen.de (A.D.); hans-ulrich.schnitzler@uni-tuebingen.de (H.-U.S.)

4 Faculty of Biology, University of Science, Vietnam National University, 334 Nguyen Trai Road, Thanh Xuan District, Hanoi 11416, Vietnam; nvsangvnu@yahoo.com (N.V.S.); huyenthucmhn@gmail.com (N.T.T.H.); hoangtrungthanh@hus.edu.vn (H.T.T.)

5 Faculty of Agro-Forestry, Tay Bac University, Chu Van An Road, Son La City 34114, Vietnam; daonhanloi@gmail.com (D.N.L.); phamvannha@utb.edu.vn (P.V.N.); ladthavong@gmail.com (L.T.)

6 Hai Duong College, 42 Nguyen Thi Due Road, Hai Duong City 03117, Vietnam; vietcdhd79@gmail.com

7 Biodiversity Research Center, Academia Sinica, No. 128, Section 2, Academia Rd., Nangang District, Taipei City 115, Taiwan; mntuanmu@gate.sinica.edu.tw (M.-N.T.); ecojoe.huang@gmail.com (J.C.-C.H.)

* Correspondence: thongvudinh@gmail.com; Tel.: +84-94-556-0108

Abstract: The Cat Ba Biosphere Reserve is internationally renowned for its spectacular karst landscape. It covers a large area with hundreds of limestone islands and various ecosystems including caves, tropical forests, and mangroves. However, previous surveys were only conducted in terrestrial ecosystems on Cat Ba Island. Therefore, bats inhabiting mangroves and the remaining islands did not receive attention from scientists up to 2014. To initially fill in the gaps, we conducted ten bat surveys between 2015 and 2020 with an emphasis on mangroves and previously unsurveyed islands. Bats were captured using mist nets and harp traps. Twenty-three species belonging to 13 genera of six families were recorded during the surveys. Of these, four species (Macroglossus minimus, Myotis hasselti, Phoniscus jagorii, Tylonycteris fulvida) are new to the reserve. Remarkably, 15 species belonging to seven genera of five families were captured in mangrove, which is the highest species diversity for bats reported from any mangrove area in mainland Southeast Asia. Based on results from the surveys and literature review, we here provide the most updated bat diversity of the reserve with confirmed records of 32 bat species belonging to 16 genera of six families. Historical records of each species in the literature were reviewed. Two species, Scotophilus heathi and Scotophilus kuhlii, are unconfirmed because of unclear evidence in previous publications. Results of this study indicated that the mangrove ecosystem is important for bats but still poorly studied in Cat Ba Biosphere Reserve and Vietnam as a whole. In addition, morphological measurements, echolocation data, distributional records, and conservation status of each species are also given in this paper for potential research and conservation campaigns in the future.

Keywords: biodiversity; Chiroptera; conservation; island; Mammalia; mangrove 


\section{Introduction}

The Cat Ba Biosphere Reserve (CBBR) is located in northern Vietnam. The reserve covers an area of 26,241 ha containing 366 limestone islands with a wide range of habitats and ecosystems [1]. Its core zone is the Cat Ba National Park which contains the largest island of CBBR, namely Cat Ba. Among the protected area systems in Vietnam, Cat Ba National Park has received the highest number of bat surveys with records included in at least 26 publications [2-27]. Of these, 19 contained bat records with references to specimens and/or relevant information sources [2-20] while remaining records were based primarily upon literature reviews [21-27] (Table 1). Before the present study, information on bat diversity of CBBR was not considered to be comprehensive because almost all previous bat surveys were conducted within the Cat Ba National Park with an emphasis on Cat Ba Island [5-13,15-17,20]. Apart from the unsurveyed islands, the mangrove areas within CBBR had not received attention from either bat experts or authorities for bat research and conservation by 2014. Although mangroves are some of the most important ecosystems on Earth and play a vital role in mitigating the negative impacts of climate change, their extent has been critically decreased by deforestation, coastal development, over-expansion of aquaculture, and other human activities [28-31]. Mangrove areas within CBBR are clearly important for biodiversity, but decreased by almost 50\% from 1990 to 2001 then continuously decreased by 15\% from 2010 to 2015 [20,31]. Between 2015 and 2020, we conducted a series of field surveys in mangroves and previously unsurveyed islands within CBBR. We also reviewed every accessible publication to provide the first confirmed species composition of bats from CBBR. This paper also provides a comprehensive background on morphological measurements, echolocation data, distributional records and conservation status of each bat species in the reserve for further research and conservation in the future.

Table 1. Bat species abundance in the study sites in CBBR.

\begin{tabular}{|c|c|c|c|c|}
\hline Study Sites & Localities & Coordinates & Habitats & Captured Species (Individuals) \\
\hline $\mathrm{A}$ & $\begin{array}{l}\text { Cai Vieng area, Phu } \\
\text { Long Commune, Cat } \\
\text { Ba Island }\end{array}$ & $\begin{array}{l}20^{\circ} 49^{\prime} 22.9^{\prime \prime} \mathrm{N} \\
106^{\circ} 54^{\prime} 40.1^{\prime \prime} \mathrm{E}\end{array}$ & Mangrove & $\begin{array}{l}\text { C. sphinx }\left(1 \sigma^{\top}\right), \text { M. minimus }(1 \text { ) }) \text {, } \\
\text { T. melanopogon }\left(2 \sigma^{\top}\right), \text { R. marshalli } \\
\left(3 \sigma^{\top}, 3 \text { \% }\right) \text {, H. larvatus }\left(2 \sigma^{\top}, 3 \text { ㅇ }\right)\end{array}$ \\
\hline B & $\begin{array}{l}\text { Cai Vieng area, Phu } \\
\text { Long Commune, Cat } \\
\text { Ba Island }\end{array}$ & $\begin{array}{l}20^{\circ} 49^{\prime} 26.4^{\prime \prime} \mathrm{N} \\
106^{\circ} 54^{\prime} 47.2^{\prime \prime} \mathrm{E}\end{array}$ & Mangrove & 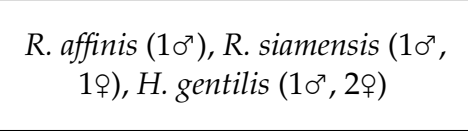 \\
\hline $\mathrm{C}$ & $\begin{array}{l}\text { Cai Vieng area, Phu } \\
\text { Long Commune, Cat } \\
\text { Ba Island }\end{array}$ & $\begin{array}{l}20^{\circ} 48^{\prime} 52.9^{\prime \prime} \mathrm{N} \\
106^{\circ} 55^{\prime} 15.7^{\prime \prime} \mathrm{E}\end{array}$ & Mangrove & $\begin{array}{l}\text { R. affinis (2o); } M \text {. hasseltii }\left(10^{\top}\right), T \text {. } \\
\text { melanopogon }\left(10^{\top}, 2 q\right)\end{array}$ \\
\hline $\mathrm{D}$ & $\begin{array}{l}\text { Cai Vieng area, Phu } \\
\text { Long Commune, Cat } \\
\text { Ba Island }\end{array}$ & $\begin{array}{l}20^{\circ} 49^{\prime} 07.4^{\prime \prime} \mathrm{N} \\
106^{\circ} 55^{\prime} 29.6^{\prime \prime} \mathrm{E}\end{array}$ & Mangrove & $\begin{array}{c}\text { R. pearsonii }\left(10^{\top}, 2 \%\right), H . \\
\text { khaokhouayensis }\left(10^{7}\right), \text { H. larvatus } \\
(1+)\end{array}$ \\
\hline $\mathrm{E}$ & $\begin{array}{l}\text { Cai Vieng area, Phu } \\
\text { Long Commune, Cat } \\
\text { Ba Island }\end{array}$ & $\begin{array}{l}20^{\circ} 49^{\prime} 53.2^{\prime \prime} \mathrm{N} \\
106^{\circ} 55^{\prime} 40.2^{\prime \prime} \mathrm{E}\end{array}$ & Mangrove & 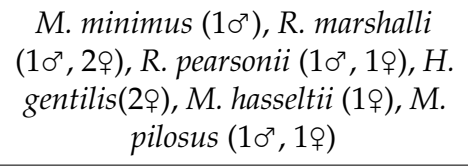 \\
\hline $\mathrm{F}$ & $\begin{array}{l}\text { Cai Vieng area, Phu } \\
\text { Long Commune, Cat } \\
\text { Ba Island }\end{array}$ & $\begin{array}{l}20^{\circ} 49^{\prime} 29.4^{\prime \prime} \mathrm{N} \\
106^{\circ} 56^{\prime} 09.5^{\prime \prime} \mathrm{E}\end{array}$ & Mangrove & $\begin{array}{c}\text { C. sphinx (1ㅇ), R. pusillus }\left(10^{7}, 1 \text { 우), }\right. \\
\text { M. alticraniatus }(19)\end{array}$ \\
\hline G & $\begin{array}{l}\text { Hoa Cuong Cave area, } \\
\text { Gia Luan Commune, } \\
\text { Cat Ba Island }\end{array}$ & $\begin{array}{l}20^{\circ} 50^{\prime} 36.8^{\prime \prime} \mathrm{N} \\
106^{\circ} 58^{\prime} 59.0^{\prime \prime} \mathrm{E}\end{array}$ & Cave and forests & 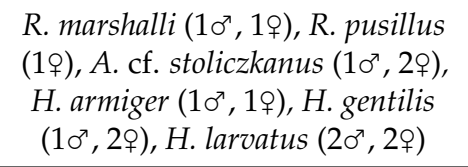 \\
\hline $\mathrm{H}$ & $\begin{array}{c}\text { Freshwater lake, } \\
\text { Headquarter area, Cat } \\
\text { Ba National Park, Cat } \\
\text { Ba Island }\end{array}$ & $\begin{array}{l}20^{\circ} 47^{\prime} 47.5^{\prime \prime} \mathrm{N} \\
106^{\circ} 59^{\prime} 41.5^{\prime \prime} \mathrm{E}\end{array}$ & Forests and plantation & 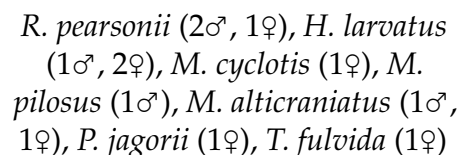 \\
\hline
\end{tabular}


Table 1. Cont.

\begin{tabular}{|c|c|c|c|c|}
\hline Study Sites & Localities & Coordinates & Habitats & Captured Species (Individuals) \\
\hline I & $\begin{array}{l}\text { Hospital Cave area, Cat } \\
\text { Ba Island }\end{array}$ & $\begin{array}{l}20^{\circ} 46^{\prime} 11.8^{\prime \prime} \mathrm{N} \\
107^{\circ} 01^{\prime} 13.7^{\prime \prime} \mathrm{E}\end{array}$ & Cave and forests & $\begin{array}{c}\text { T. melanopogon }\left(2 \sigma^{\top}\right), \text { A. cf. } \\
\text { stoliczkanus }(3+\text { ) }) \text { H. alongensis } \\
(1+), \text { H. pulveratus }\left(1 \sigma^{\top}, 1 \text { 우), P. }\right. \\
\text { abramus }\left(10^{\top}\right)\end{array}$ \\
\hline $\mathrm{J}$ & $\begin{array}{l}\text { Viet Hai Cave area, Viet } \\
\text { Hai Commune, Cat Ba } \\
\text { Island }\end{array}$ & $\begin{array}{l}20^{\circ} 48^{\prime} 08.0^{\prime \prime} \mathrm{N} \\
107^{\circ} 02^{\prime} 12.5^{\prime \prime} \mathrm{E}\end{array}$ & Cave and forests & $\begin{array}{c}\text { H. alongensis }\left(10^{7}\right), \text { H. larvatus }\left(10^{7} \text {, }\right. \\
\text { 19), H. pulveratus (2\%) }\end{array}$ \\
\hline K & $\begin{array}{l}\text { Monkey Island, Cat Ba } \\
\text { National Park }\end{array}$ & $\begin{array}{l}20^{\circ} 46^{\prime} 11.1^{\prime \prime} \mathrm{N} \\
107^{\circ} 05^{\prime} 03.2^{\prime \prime} \mathrm{E}\end{array}$ & Forests and shrubs & $\begin{array}{l}\text { H. larvatus }\left(1 \sigma^{\pi}, 19\right), \text { H. pulveratus } \\
\left(2 \sigma^{\pi}, 2+\right)\end{array}$ \\
\hline $\mathrm{L}$ & $\begin{array}{c}\text { An unnamed island, } \\
\text { Cat Ba Biosphere } \\
\text { Reserve }\end{array}$ & $\begin{array}{l}20^{\circ} 46^{\prime} 17.7^{\prime \prime} \mathrm{N} \\
107^{\circ} 05^{\prime} 52.7^{\prime \prime} \mathrm{E}\end{array}$ & Forests and shrubs & $\begin{array}{c}\text { R. affinis }\left(10^{\top}, 2 \%\right), \text { R. marshalli } \\
\text { (1우), R. pusillus }\left(1 \sigma^{\top}\right) ; \text { A. cf. } \\
\text { stoliczkanus (1), H. larvatus (1), } \\
\text { M. fuliginosus (1ㅇ) }\end{array}$ \\
\hline M & $\begin{array}{c}\text { An unnamed island, } \\
\text { Cat Ba Biosphere } \\
\text { Reserve }\end{array}$ & $\begin{array}{l}20^{\circ} 51^{\prime} 47.6^{\prime \prime} \mathrm{N} \\
106^{\circ} 58^{\prime} 29.0^{\prime \prime} \mathrm{E}\end{array}$ & Mangrove and forests & $\begin{array}{c}\text { H. larvatus (19), H. pulveratus }\left(2 \sigma^{7},\right. \\
2 \text {, }), \text { M. cyclotis }\left(1 \sigma^{7}\right), \text { M. pilosus } \\
\text { (1\%) }\end{array}$ \\
\hline $\mathrm{N}$ & $\begin{array}{l}\text { Hien Hao Commune, } \\
\text { Cat Ba Island }\end{array}$ & $\begin{array}{l}20^{\circ} 47^{\prime} 10.1^{\prime \prime} \mathrm{N} \\
106^{\circ} 58^{\prime} 08.0^{\prime \prime} \mathrm{E}\end{array}$ & Forests and plantation & 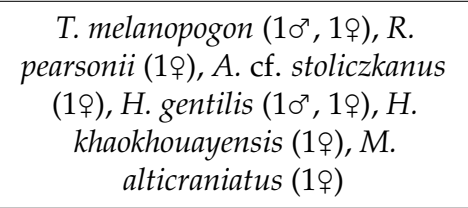 \\
\hline $\mathrm{O}$ & $\begin{array}{c}\text { An unnamed island, } \\
\text { Cat Ba Biosphere } \\
\text { Reserve }\end{array}$ & $\begin{array}{l}20^{\circ} 45^{\prime} 45.4^{\prime \prime} \mathrm{N} \\
107^{\circ} 03^{\prime} 35.2^{\prime \prime} \mathrm{E}\end{array}$ & Forests and shrubs & $\begin{array}{l}\text { R. siamensis }\left(2 \sigma^{7}, 2 \circ\right), \text { H. armiger } \\
\left(1 \sigma^{\top}\right), H . \text { pulveratus }\left(1 \sigma^{\top}\right)\end{array}$ \\
\hline
\end{tabular}

\section{Materials and Methods}

\subsection{Bat Capture and Morphological Measurements}

Ten bat surveys were conducted at fifteen sites within CBBR between May 2015 and October 2020 (Figure 1; Table 1). Bats were captured and handled following guidelines recommended by the American Society of Mammalogists [32,33]. Three four-bank harp traps [34] and six mist nets of various sizes (height $2.6 \mathrm{~m}$, length 3-12 $\mathrm{m}$, and mesh size $16 \times 16 \mathrm{~mm}$ ) were set up across footpaths or narrow streams under the forest canopy as well as in the channels in mangroves to capture bats. Each captured bat was removed carefully from the trap or net and held individually in cotton bags. The following external measurements were taken using a digital caliper (ABSOLUTE Coolant Proof Caliper Series 500, Mitutoyo America Corporation, Illinois 60502, IL, USA) to the nearest $0.1 \mathrm{~mm}$ : FA, forearm length-from the extremity of the elbow to the extremity of the carpus with the wings folded; EH, ear height-length of ear conch; EW, ear width-the greatest width of ear conch; NL, anterior nose-leaf width — the greatest width of the anterior leaf; Tail, tail lengthfrom the anal opening to the tip of the tail; TIB, tibia length-from the knee joint to the ankle; $\mathrm{HF}$, hind-foot length-from the extremity of the heel behind the os calcis to the extremity of the longest digit, excluding the hairs or claws. Selected bats were kept as specimens for confirmed identification in museums following the protocol code 751/STTNSV approved on 26 August 2015 by the Institute of Ecology and Biological Resources. Their skulls were extracted and cleaned for the following craniodental measurements: SL, total length of skull - from occiput to the most anterior part of the canine; CCL, condylocanine length-from the exoccipital condyle to the most anterior part of the canine; IOW, interorbital width-the least width of the interorbital constriction; ZW, zygomatic width-the greatest width of the skull across the zygomatic arches; MW, mastoid width-the greatest distance across the mastoid region; $\mathrm{C} 1-\mathrm{C} 1$, width across the upper canines-greatest width, taken across the outer borders of upper canines; M3-M3, width across the upper molars-greatest width, 
taken across the outer crowns of the last upper molars; $\mathrm{ml}$, mandible length-from the anterior rim of the alveolus of the 1st lower incisor to the most posterior part of the condyle; c1-m3, mandibular tooth row length-from the front of the lower canine to the back of the crown of the 3 rd lower molar. The above methods and measurements are described in Thong et al. [12,13] and illustrated in Bates and Harrison [35] and Csorba et al. [36]. Reproductive status and age were assessed following Racey [37] and Brunet-Rossinni and Wilkinson [38], respectively.

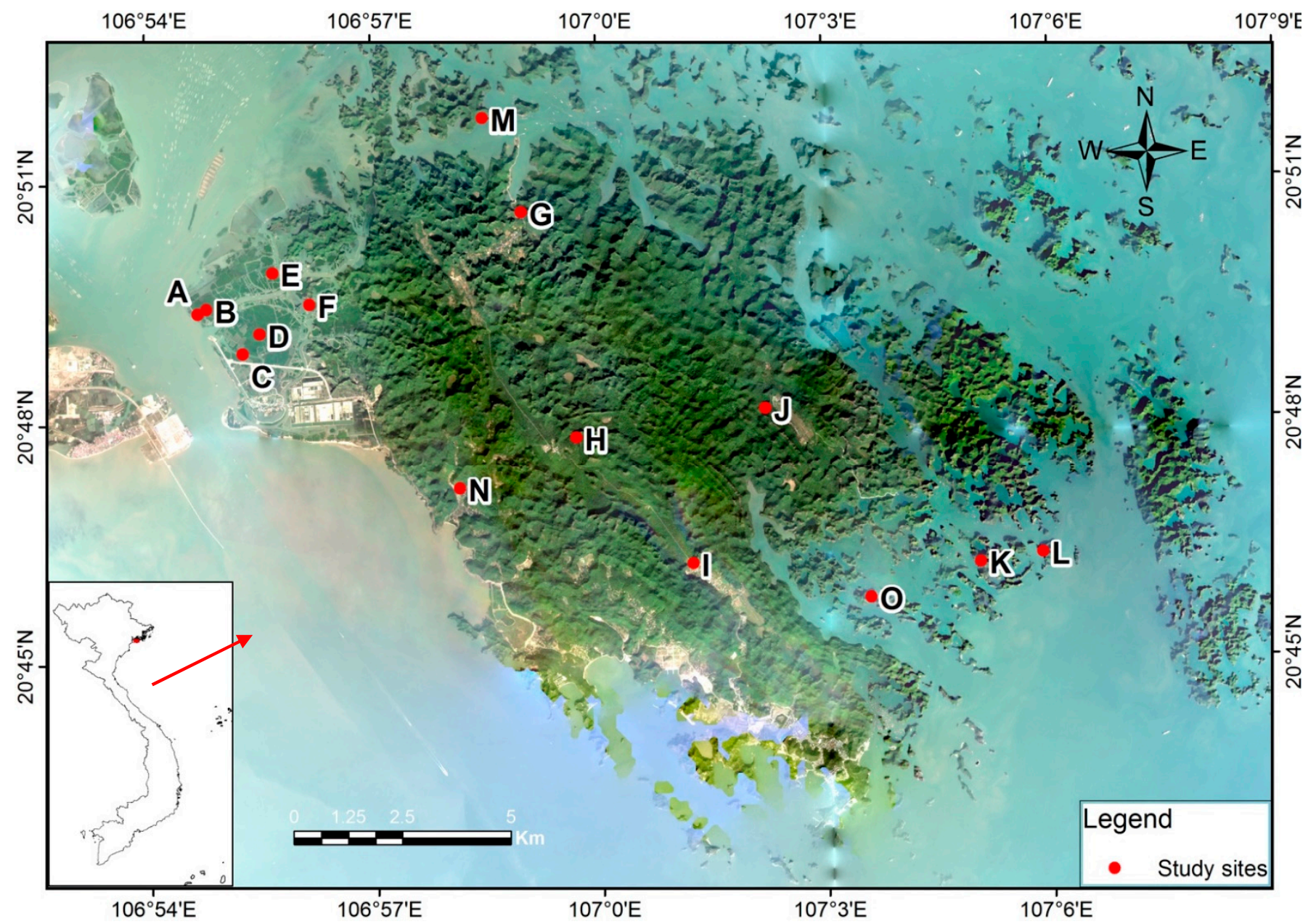

Figure 1. Map of Cat Ba Biosphere Reserve. The solid red dots and capital letters represent the study sites between 2015 and 2020. The local name and geographical coordinate of each site are given in Table 1. Background map was obtained from the "Satellite imagery: Planet Labs Inc. (San Francisco, CA, USA)".

\subsection{Recording and Analysis of Echolocation Signals}

Echolocation calls of 84 captured echolocating bats were recorded when each of them was flying inside a flight tent $(5 \mathrm{~m}$ (length) $\times 5 \mathrm{~m}$ (width) $\times 3 \mathrm{~m}$ (height)) using a PCTape system. Batman software was used to detect high-quality calls before recording. Additional calls were recorded when bats were released in the field. Selected recordings were analyzed using Selena software. The PCTape system, Batman and Selena software are custom-made by the Department of Animal Physiology, Faculty of Sciences, University of Tübingen (Tübingen, Germany). Species of Emballonuridae, Vespertilionidae, and Miniopteridae are regarded as "FM bats" because they emit "frequency-modulated" (FM) calls. Species of rhinolophid (Rhinolophidae) and hipposiderid (Hipposideridae) bats are regarded as " $\mathrm{CF}$ bats" because they emit long multiharmonic echolocation calls containing a dominant "constant frequency" (CF) component. Each entire harmonic of rhinolophid species comprises three components: initial frequency-modulated (iFM), constant frequency $(\mathrm{CF})$, and termi- 
nal frequency-modulated (tFM). Each entire harmonic of hipposiderid species comprises two components: CF and tFM. The first harmonic of FM bats and the second harmonic of CF bat species contain the maximum energy of each call. Therefore, we measured three sound parameters of the first harmonic of FM bats (iFM, tFM, and pulse duration) and two sound parameters of the second harmonic of CF bats (CF2 and pulse duration).

\subsection{Tissue Sampling and Genetic Analysis}

Samples for genetic analyses in this study comprised wing biopsies from alive individuals, which were released in the field after identification, and tissues from voucher specimens preserved in 100\% ethanol at the Institute of Ecology and Biological Resources and the VNU University of Sciences (Hanoi, Vietnam). Total DNA was isolated from samples using DNeasy Blood \& Tissue Kits (Qiagen, Valencia, CA, USA) following the manufacturer's recommendations. The $657 \mathrm{bp}$ target fragment of COI gene was amplified using the universal primer pair for mammals VF1d: 5' - TTC TCA ACC AAC CAC AAR GAY ATY GG -3'; VR1d: 5' - TAG ACT TCT GGG TGG CCR AAR AAY CA -3' [39]. The PCR reactions were carried out with components of $1 x$ Phusion PCR buffer, $0.3 \mu \mathrm{M}$ each primer (VF1d and VR1d), 0.2 mM dNTP mix, 1 U Phusion enzyme (Thermo Scientific, Waltham, MA, USA), and $10 \mathrm{ng}$ DNA template. The thermal cycle condition was set up as follow: initial denaturation at $95^{\circ} \mathrm{C}$ for $2 \mathrm{~min}, 35$ cycles of denaturation at $95^{\circ} \mathrm{C}$ for $20 \mathrm{~s}$, and annealing at $50-57^{\circ} \mathrm{C}$ for $20 \mathrm{~s}$ and an extension at $72{ }^{\circ} \mathrm{C}$ for $30 \mathrm{~s}$. A final extension was run at $72{ }^{\circ} \mathrm{C}$ for $5 \mathrm{~min}$. The PCR products were purified with MEGAquick-spin ${ }^{\mathrm{TM}}$ kit (iNtRON Biotechnology, Gyeonggi-do, South Korea) and sequenced in both directions using the BigDye Terminator v.3.1 Cycle Sequencing Kit (1st BASE, Selangor, Malaysia). The sequencing results were analyzed using BioEdit software [40]. Analysis of molecular phylogeny based on the aligned COI sequence data was performed by MEGA7 software [41]. Phylogenetic relationships were estimated based on the Neighbor-Joining method [42]. A distancebased tree was built with the Neighbour-Joining algorithm using the Kimura-2-parameter model [43]. Branch support was assessed by 1000 bootstrap replicates.

\section{Results}

A total of 123 bats were captured at 15 study sites over the surveys, representing 23 species belonging to 13 genera of six families (Tables 1-3): Cynopterus sphinx, Macroglossus minimus, Taphozous melanopogon, Rhinolophus affinis, R. siamensis, R. marshalli, R. pearsonii, R. pusillus, Aselliscus cf. cf. stoliczkanus, Hipposideros alongensis, H. armiger, H. gentilis, $H$. larvatus, H. khaokhouayensis, Hypsugo pulveratus, Murina cyclotis, Myotis hasseltii, M. pilosus, M. alticraniatus, Phoniscus jagorii, Pipistrellus abramus, Tylonycteris fulvida and Miniopterus fuliginosus. Their morphological features are similar to descriptions in previous publications which are cited in each species account of the following Discussion section. The measurements of captured bats in CBBR did not exhibit any sexual dimorphism. Four species were common throughout the study sites (R. marshalli, H. gentilis, H. larvatus, and H. pulveratus), and four species are new to CBBR (M. minimus, M. hasselti, P. jagorii, and T. fulvida). Fifteen species belonging to seven genera of five families were captured in mangroves, including Pteropodidae (C. sphinx, M. minimus), Emballonuridae (T. melanopogon), Rhinolophidae (R. affinis, R. siamensis, R. marshalli, R. pearsonii, R. pusillus), Hipposideridae (Hipposideros larvatus, H. gentilis, H. khoukhouayensis), and Vespertilionidae (H. pulveratus, M. hasseltii, M. pilosus, M. alticraniatus). M. minimus and M. hasselti were only recorded in the mangrove ecosystem while the remaining species were recorded in different habitats including mangroves, caves, forests, and plantations. Our finding of M. minimus does not only represent a new species record to CBBR but also the first confirmed occurrence in northern Vietnam. Among the 21 echolocating species, we analyzed echolocation calls of 20 species. The values of iFM, CF2, tFM, and pulse duration of each species are given in the respective species account. 
Table 2. Seven external measurements (in mm) of bat species recorded from Cat Ba Biosphere Reserve between 2015 and 2020. Abbreviations are defined in the Material and Methods section. Sample sizes differing from those reported under $n$ are given in paratheses. Data are presented as a range (minimum and maximum) and mean and standard deviation are included when the sample size is $\geq 3$.

\begin{tabular}{|c|c|c|c|c|c|c|c|c|}
\hline Species & $\mathbf{n}$ & FA & EH & EW & NL & Tail & ТIB & HF \\
\hline Cynopterus sphinx & 2 & $82.3 ; 83.6$ & $16.8 ; 19.5$ & - & - & $6.8 ; 8.3$ & $26.6 ; 36.2$ & $14.5 ; 16.5$ \\
\hline $\begin{array}{l}\text { Macroglossus } \\
\text { minimus }\end{array}$ & 2 & $42.2 ; 44.2$ & $12.7 ; 13.1$ & $8.6 ; 9.9$ & - & - & $16.4 ; 17.6$ & $10.0 ; 10.5$ \\
\hline $\begin{array}{l}\text { Taphozous } \\
\text { melanopogon }\end{array}$ & 9 & $\begin{array}{c}65.7 \pm 1.4 \\
63.7-67.3\end{array}$ & $\begin{array}{l}16.7 \pm 4.4 \\
11.5-21.3\end{array}$ & $\begin{array}{l}11.7 \pm 1.0 \\
10.5-13.3\end{array}$ & - & $\begin{array}{l}22.9 \pm 1.2 \\
20.7-24.6\end{array}$ & $\begin{array}{l}25.1 \pm 0.8 \\
23.1-25.9\end{array}$ & $\begin{array}{l}10.4 \pm 0.3 \\
10.0-10.8\end{array}$ \\
\hline Rhinolophus affinis & 6 & $\begin{array}{c}51.4 \pm 0.9 \\
50.4-52.5\end{array}$ & $\begin{array}{l}21.3 \pm 0.8 \\
20.0-22.0\end{array}$ & $\begin{array}{l}16.3 \pm 0.9 \\
15.0-17.5\end{array}$ & - & - & - & - \\
\hline R. siamensis & 6 & $\begin{array}{l}38.8 \pm 0.7 \\
37.7-39.6\end{array}$ & $\begin{array}{l}20.2 \pm 0.5 \\
19.9-21.0\end{array}$ & $\begin{array}{l}14.3 \pm 1.3 \\
12.5-15.6\end{array}$ & - & - & - & - \\
\hline R. marshalli & 12 & $\begin{array}{c}46.5 \pm 1.5 \\
44.4-48.6\end{array}$ & $\begin{array}{l}24.9 \pm 1.4 \\
23.1-26.8\end{array}$ & $\begin{array}{l}17.3 \pm 0.8 \\
15.8-18.3\end{array}$ & $\begin{array}{c}6.6 \pm 2.7 \\
4.7-8.5(2)\end{array}$ & $\begin{array}{c}19.6 \pm 0.5 \\
19.4-20.2(3)\end{array}$ & $\begin{array}{c}18.8 \pm 1.1 \\
17.6-19.8(3)\end{array}$ & $\begin{array}{c}6.5 \pm 1.2 \\
5.1-7.2(3)\end{array}$ \\
\hline R. pearsonii & 9 & $\begin{array}{c}51.9 \pm 1.3 \\
49.0-53.4\end{array}$ & $\begin{array}{l}23.4 \pm 1.5 \\
21.5-26.0\end{array}$ & $\begin{array}{c}16.9,1.1 \\
14.1-18.0\end{array}$ & $\begin{array}{c}11.9 \pm 0.3 \\
11.7-12.3(3)\end{array}$ & $\begin{array}{c}20.1 \pm 2.1 \\
17.7-21.8(3)\end{array}$ & $\begin{array}{c}25.9 \pm 1.2 \\
24.5-26.9(3)\end{array}$ & $\begin{array}{c}10.5 \pm 0.3 \\
10.3-10.9(3)\end{array}$ \\
\hline R.pusillus & 4 & $\begin{array}{l}36.1 \pm 1.5 \\
34.0-37.5\end{array}$ & $\begin{array}{l}16.1 \pm 0.8 \\
15.0-16.8\end{array}$ & $\begin{array}{c}11.3 \pm 1.0 \\
9.8-12.0\end{array}$ & $7.2 ; 7.5$ & $18.0 ; 20.1(2)$ & $15.0 ; 15.8(2)$ & $4.1 ; 6.0(2)$ \\
\hline $\begin{array}{l}\text { Aselliscus cf. } \\
\text { stoliczkanus }\end{array}$ & 8 & $\begin{array}{c}44,6 \pm 1.5 \\
41.3-44.6\end{array}$ & $\begin{array}{c}9.5 \pm 0.6 \\
8.5-9.5\end{array}$ & $\begin{array}{c}8.7 \pm 0.4 \\
8.4-8.7(3)\end{array}$ & $\begin{array}{c}5.5 \pm 0.2 \\
5.4-5.5(3)\end{array}$ & $\begin{array}{c}35.8 \pm 4.1 \\
33.3-35.8(4)\end{array}$ & $\begin{array}{c}19.5 \pm 0.5 \\
19.1-19.5(4)\end{array}$ & $\begin{array}{c}4.7 \pm 0.5 \\
4.3-4.7(4)\end{array}$ \\
\hline $\begin{array}{l}\text { Hipposideros } \\
\text { alongensis }\end{array}$ & 2 & $69.68 ; 70.48$ & $23.36 ; 23.84$ & - & - & - & - & - \\
\hline H. armiger & 3 & $\begin{array}{l}94.3 \pm 2.0 \\
92.1-95.7\end{array}$ & $\begin{array}{l}26.9 \pm 4.0 \\
22.5-30.4\end{array}$ & $23.22(1)$ & $12.09(1)$ & $30.11(1)$ & $40.5(1)$ & $15.16(1)$ \\
\hline H. gentilis & 10 & $\begin{array}{l}41.5 \pm 1.4 \\
38.2-43.4\end{array}$ & $\begin{array}{l}23.0 \pm 2.3 \\
20.1-27.4\end{array}$ & $\begin{array}{c}17.1 \pm 1.6 \\
14.5-19.3(9)\end{array}$ & $\begin{array}{l}5.4 \pm 0.7 \\
4.3-6.6(9)\end{array}$ & $\begin{array}{c}29.0 \pm 4.4 \\
23.4-36.0(9)\end{array}$ & $\begin{array}{c}19.5 \pm 0.4 \\
19.0-20.1(9)\end{array}$ & $\begin{array}{c}5.2 \pm 1.1 \\
3.9-6.6(9)\end{array}$ \\
\hline H. larvatus & 19 & $\begin{array}{l}54.9 \pm 2.0 \\
48.7-59.0\end{array}$ & $\begin{array}{l}20.4 \pm 2.2 \\
14.4-23.5\end{array}$ & $\begin{array}{c}16.2 \pm 1.3 \\
14.4-17.9(12)\end{array}$ & $\begin{array}{c}7.1 \pm 0.9 \\
5.3-8.3(12)\end{array}$ & $\begin{array}{c}30.5 \pm 3.2 \\
24.8-35.5(12)\end{array}$ & $\begin{array}{c}21.7 \pm 0.9 \\
20.2-22.6(12)\end{array}$ & $\begin{array}{c}8.5 \pm 0.8 \\
6.8-10.1(12) \\
\end{array}$ \\
\hline H. khaokhouayensis & 2 & $43.1 ; 42.2$ & $22.4 ; 20.6$ & $17.0(1)$ & $5.5(1)$ & $28.2(1)$ & $28.2(1)$ & $6.4(1)$ \\
\hline Hypsugo pulveratus & 13 & $\begin{array}{l}35.2 \pm 0.7 \\
33.7-36.3\end{array}$ & $\begin{array}{c}10.6 \pm 1.3 \\
8.9-12.8\end{array}$ & $\begin{array}{c}7.7 \pm 0.6 \\
6.7-8.2\end{array}$ & - & $\begin{array}{c}34.2 \pm 0.4 \\
33.8-34.5(5)\end{array}$ & $\begin{array}{c}14.5 \pm 0.2 \\
14.3-14.7(5)\end{array}$ & $\begin{array}{c}6.0 \pm 0.1 \\
5.8-6.1(5)\end{array}$ \\
\hline Murina cyclotis & 2 & $31.3 ; 34.1$ & $14.7 ; 13.9$ & $9.3 ; 9.9$ & & $36.0 ; 38.8$ & $16.8 ; 19.0$ & $7.9 ; 7.8$ \\
\hline Myotis alticraniatus & 4 & $\begin{array}{l}33.6 \pm 1.3 \\
32.1-35.1\end{array}$ & $\begin{array}{l}12.9 \pm 3.3 \\
10.0-15.9\end{array}$ & $\begin{array}{c}5.4 \pm 0.3 \\
5.0-5.8\end{array}$ & - & $\begin{array}{l}18.0 \pm 2.2 \\
15.6-20.3\end{array}$ & $\begin{array}{l}14.0 \pm 0.5 \\
13.5-14.7\end{array}$ & $\begin{array}{c}5.2 \pm 0.5 \\
4.6-5.9\end{array}$ \\
\hline M. hasseltii & 2 & $38.6 ; 38.5$ & $13.5 ; 14.3$ & - & - & $40.0 ; 38.5$ & $16.8 ; 15.6$ & $9.8 ; 10.5$ \\
\hline M. pilosus & 4 & $\begin{array}{c}54.0 \pm 3.0 \\
51.1-56.7\end{array}$ & $\begin{array}{l}17.2 \pm 0.9 \\
16.2-18.3\end{array}$ & $\begin{array}{c}13.8 \pm 2.9 \\
10.5-16.2\end{array}$ & - & - & $\begin{array}{l}20.5 \pm 1.1 \\
18.9-21.6\end{array}$ & $\begin{array}{l}16.0 \pm 2.3 \\
13.4-18.9\end{array}$ \\
\hline Phoniscus jagorii & 1 & 35.8 & 13.4 & 9.7 & & 36.4 & 16.7 & 9.3 \\
\hline Pipistrellus abramus & 1 & 28.6 & 12.5 & - & - & 38.6 & - & - \\
\hline Tylonycteris fulvida & 1 & 26.8 & 8.8 & 5.8 & & 32.3 & 11.2 & 8.8 \\
\hline $\begin{array}{l}\text { Miniopterus } \\
\text { fuliginosus }\end{array}$ & 1 & 52.1 & 8.9 & 8.4 & - & 34.1 & 21.4 & 10.0 \\
\hline
\end{tabular}


Table 3. Ten craniodental measurements (in mm) of bat species recorded from Cat Ba Biosphere Reserve between 2015 and 2020. Abbreviations are defined in the Material and Methods section. Sample sizes differing from those reported under $n$ are given in paratheses. Data are presented as a range, mean and SD are included when the sample size is $\geq 3$.

\begin{tabular}{|c|c|c|c|c|c|c|c|c|c|c|}
\hline Species & $\mathbf{n}$ & SL & CCL & IOW & ZW & MW & $\mathrm{C} 1-\mathrm{C} 1$ & M3-M3 & $\mathrm{mL}$ & c1-m3 \\
\hline $\begin{array}{l}\text { Taphozous } \\
\text { melanopogon }\end{array}$ & 9 & $\begin{array}{c}21.6 \pm 0.2 \\
21.3-21.8\end{array}$ & $\begin{array}{c}20.0 \pm 0.4 \\
19.4-20.6\end{array}$ & $\begin{array}{c}4.9 \pm 0.2 \\
4.5-5.2\end{array}$ & $\begin{array}{l}12.4 \pm 0.3 \\
11.8-12.6\end{array}$ & $\begin{array}{l}11.1 \pm 0.1 \\
11.0-11.3\end{array}$ & $\begin{array}{c}3.8 \pm 0.2 \\
3.5-4.1\end{array}$ & $\begin{array}{c}8.8 \pm 0.1 \\
8.6-8.9\end{array}$ & $\begin{array}{l}16.0 \pm 0.2 \\
15.6-16.3\end{array}$ & $\begin{array}{c}9.8 \pm 0.2 \\
9.6-10.1\end{array}$ \\
\hline $\begin{array}{l}\text { Rhinolophus } \\
\text { marshalli }\end{array}$ & 3 & $\begin{array}{l}19.1 \pm 0.5 \\
18.7-19.6\end{array}$ & $\begin{array}{l}17.3 \pm 0.6 \\
16.7-17.9\end{array}$ & $\begin{array}{l}2.4 \pm 0 \\
2.4-2.5\end{array}$ & $\begin{array}{c}8.3 \pm 0.1 \\
8.2-8.4\end{array}$ & $\begin{array}{c}9.1 \pm 0.3 \\
8.8-9.4\end{array}$ & $\begin{array}{c}3.7 \pm 0.3 \\
3.3-3.9\end{array}$ & $\begin{array}{c}5.4 \pm 0,1 \\
5.4-5.5\end{array}$ & $\begin{array}{l}11.3 \pm 0.3 \\
11.0-11.6\end{array}$ & $\begin{array}{c}6.6 \pm 0.2 \\
6.4-6.8\end{array}$ \\
\hline R. pearsonii & 3 & $\begin{array}{l}24.2 \pm 0.2 \\
24.0-24.3\end{array}$ & $\begin{array}{l}21.9 \pm 0.3 \\
21.6-22.1\end{array}$ & $\begin{array}{c}2.3 \pm 0.1 \\
2.2-2.4\end{array}$ & $\begin{array}{l}11.1 \pm 0.1 \\
11.0-11.3\end{array}$ & $\begin{array}{l}10.4 \pm 0.2 \\
10.2-10.6\end{array}$ & $\begin{array}{c}5.9 \pm 0.1 \\
5.8-6.1\end{array}$ & $\begin{array}{c}8.3 \pm 0.1 \\
8.2-8.3\end{array}$ & $\begin{array}{l}16.0 \pm 0.3 \\
15.7-16.4\end{array}$ & $\begin{array}{c}9.9 \pm 0.2 \\
9.7-10.1\end{array}$ \\
\hline R. pusillus & 2 & $15.6 ; 15.7$ & $14.2 ; 14.6$ & $2.06 ; 2.1$ & $7.1 ; 7.4$ & $7.2 ; 7.3$ & $3.2 ; 3.7$ & $5.2 ; 5.3$ & $9.6 ; 10.0$ & 5.67 \\
\hline $\begin{array}{l}\text { Aselliscus cf. } \\
\text { stoliczkanus }\end{array}$ & 4 & $\begin{array}{c}15.5 \pm 0.3 \\
15.1-16.0\end{array}$ & $\begin{array}{c}13.9 \pm 0.3 \\
13.6-14.2\end{array}$ & $\begin{array}{c}2.0 \pm 0.1 \\
1.9-2.1\end{array}$ & $\begin{array}{c}7.6 \pm 0.1 \\
7.5-7.7\end{array}$ & $\begin{array}{c}7.3 \pm 0.1 \\
7.3-7.4\end{array}$ & $\begin{array}{c}3.3 \pm 0.3 \\
3.1-3.7\end{array}$ & $\begin{array}{c}5.4 \pm 0.1 \\
5.3-5.5\end{array}$ & $\begin{array}{c}9.5 \pm 0.3 \\
9.3-9.8\end{array}$ & $\begin{array}{c}5.5 \pm 0.4 \\
5.0-5.8\end{array}$ \\
\hline $\begin{array}{l}\text { Hipposideros } \\
\text { armiger }\end{array}$ & 1 & 32.33 & 28.48 & 4.22 & 17.82 & 15.34 & 8.8 & 12.24 & 22.42 & 13.6 \\
\hline H. gentilis & 9 & $\begin{array}{l}18.4 \pm 0.6 \\
17.7-19.2\end{array}$ & $\begin{array}{c}15.9 \pm 0.3 \\
5.6-16.4\end{array}$ & $\begin{array}{c}2.7 \pm 0.1 \\
2.6-2.9\end{array}$ & $\begin{array}{c}8.9 \pm 0.4 \\
8.4-9.9\end{array}$ & $\begin{array}{c}9.5,0.7 \\
8.9-10.5\end{array}$ & $\begin{array}{c}3.5 \pm 0.1 \\
3.3-3.7\end{array}$ & $\begin{array}{c}6.5 \pm 0.5 \\
5.7,7.0\end{array}$ & $\begin{array}{c}10.9 \pm 0.2 \\
10.7,11.0\end{array}$ & $\begin{array}{c}6.6 \pm 0.2 \\
6.4-6.8\end{array}$ \\
\hline H. larvatus & 12 & $\begin{array}{c}21.6 \pm 0.3 \\
21.2-21.8\end{array}$ & $\begin{array}{c}19.5 \pm 0.7 \\
18.4-2.0\end{array}$ & $\begin{array}{c}3.1 \pm 0.2 \\
2.9-3.3\end{array}$ & $\begin{array}{l}12.2 \pm 0.4 \\
12.0-12.8\end{array}$ & $\begin{array}{l}10.3 \pm 0.2 \\
10.1-10.5\end{array}$ & $\begin{array}{c}4.7 \pm 0.2 \\
4.5-4.9\end{array}$ & $\begin{array}{c}8.2 \pm 0.2 \\
8.0-8.4\end{array}$ & $\begin{array}{l}14.4 \pm 0.3 \\
14.2-14.7\end{array}$ & $\begin{array}{c}8.8 \pm 0.1 \\
8.7-9.0\end{array}$ \\
\hline H. khaokhouayensis & 1 & 17.88 & 16.22 & 2.55 & 8.5 & 9.36 & 3.62 & 5.6 & 10.77 & 6.18 \\
\hline $\begin{array}{c}\text { Myotis } \\
\text { alticraniatus }\end{array}$ & 4 & $\begin{array}{l}12.2 \pm 0.3 \\
11.8-12.5\end{array}$ & $\begin{array}{l}11.0 \pm 0.6 \\
10.5-11.7\end{array}$ & $\begin{array}{c}3.1 \pm 0.2 \\
3.0-3.3\end{array}$ & $\begin{array}{c}7.3 \pm 0.3 \\
7.0-7.7\end{array}$ & $\begin{array}{l}6.5,0.1 \\
6.4-6.7\end{array}$ & $\begin{array}{c}2.2 \pm 0.1 \\
2.2-2.3\end{array}$ & $\begin{array}{c}4.9 \pm 0.1 \\
4.7-5.0\end{array}$ & $\begin{array}{c}8.5 \pm 0.1 \\
8.4-8.7\end{array}$ & $\begin{array}{c}4.2 \\
4.2-4.3\end{array}$ \\
\hline M. pilosus & 1 & 20.09 & 19.04 & 4.9 & 12.5 & 9.66 & 5.44 & 8.18 & 15.13 & 8.15 \\
\hline $\begin{array}{l}\text { Miniopterus } \\
\text { fuliginosus }\end{array}$ & 1 & 16.92 & 16.08 & 4.08 & 9.58 & 9.17 & 3.35 & 7.32 & 11.68 & 7.2 \\
\hline
\end{tabular}

\section{Discussion}

To date, 32 bat species belonging to 16 genera and six families are known from CBBR (Table 4). This is the first confirmed bat species composition in the reserve based on historical records and results from our results between 2015 and 2020. Of these, 27 species were listed in previous publications (Table 4). Records of two species, Scotophilus heathi and Scotophilus kuhlii, were first reported by Canh et al. [21] and cited in four subsequent publications $[9,23,24,27]$. However, these records were not referred to any specimen or information sources. Therefore, their presence in CBBR is not confirmed and they are not accounted for in the current species composition of CBBR. With fifteen species belonging to seven genera of five families recorded in the mangrove, this species composition not only exhibits the highest species diversity for bats reported from any mangrove area in mainland Southeast Asia but also indicates the importance of the mangrove ecosystem for bat research and conservation. Unfortunately, mangrove in Vietnam has been threatened and declined due to aquaculture expansion, infrastructure developments, and other factors [31]. Within CBBR, the mangrove area was declined by almost $50 \%$ between 1990 and 2001 while the remaining area has continuously declined by $15 \%$ between 2010 and 2015 [31]. Bats of CBBR have likely been negatively threatened by habitat loss and other factors. Four species (Coelops frithii, Harpiocephalus harpia, Myotis muricola, and Pipistrellus javanicus) and five species (Cynopterus horsfieldii, Rousettus amplexicaudatus, Hipposideros griffini and Murina harrisoni) have not been recorded since 2002 and 2014, respectively (Table 4). The CF2 values of each CF bat species in CBBR are distinct and can be used for acoustic identification in the field. The iFM and tFM values of FM bats in the reserve are in a wide variation. 
Table 4. A confirmed bat species composition in Cat Bat Biosphere Reserve, Northeastern Vietnam, based on data from the 2015-2020 surveys and literature review.

\begin{tabular}{|c|c|c|c|}
\hline Scientific Name & Common Name & $\begin{array}{c}\text { Records with Reference to } \\
\text { Material(s) }\end{array}$ & $\begin{array}{l}\text { Recorded without Reference to } \\
\text { Material(s) }\end{array}$ \\
\hline Pteropodidae & Fruit bats & & \\
\hline Cynopterus sphinx & Greater short-nosed fruit bat & $\begin{array}{l}\text { Abramov \& Kruskop } 2012 \text { [9]; } \\
\text { Furey } 2002 \text { [20]; This study }\end{array}$ & $\begin{array}{l}\text { Canh et al. } 1997 \text { [21]; Thong } 2008 \text { [23]; } \\
\text { Thong \& Furey } 2008 \text { [24]; Can et al. } 2008 \\
\text { [25]; Thong et al. 2020 [27] }\end{array}$ \\
\hline C. horsfieldii & Horsfield's fruit bat & Thong 2014b [17] & Thong et al. 2020 [27] \\
\hline Macroglossus minimus & $\begin{array}{l}\text { Dagger-toothed long-nosed fruit } \\
\text { bat }\end{array}$ & This study & \\
\hline Rousettus amplexicaudatus & Geoffroy's rousette & Abramov \& Kruskop 2012 [9]; & $\begin{array}{c}\text { Kruskop } 2013 \text { [14]; Thong } 2008 \text { [23]; } \\
\text { Thong et al. } 2020 \text { [27] }\end{array}$ \\
\hline Emballonuridae & Sheath-tailed and tomb bats & & \\
\hline Taphozous melanopogon & Black-bearded tomb bat & Thong et al. 2016 [18]; This study & $\begin{array}{c}\text { Abramov \& Kruskop } 2012 \text { [9]; Kruskop } \\
2013 \text { [14]; Borrisenko\&Kruskop } 2003 \\
\text { [22]; Can et al. } 2008 \text { [25]; Nga \& Tung } \\
\text { 2018 [26]; Thong et al. } 2020 \text { [27] }\end{array}$ \\
\hline Rhinolophidae & Horseshoe bats & & \\
\hline Rhinolophus affinis & Intermediate horseshoe bat & $\begin{array}{l}\text { Thong } 2011 \text { [7]; Thong, 2014a [16]; } \\
\text { This study }\end{array}$ & $\begin{array}{c}\text { Abramov \& Kruskop } 2012 \text { [9]; Thong } \\
2008 \text { [23]; Thong \& Furey 2008 [24]; } \\
\text { Thong et al. 2020 [27] }\end{array}$ \\
\hline R. siamensis & Thai horseshoe bat & $\begin{array}{c}\text { Thong } 2011[7] \text { (=R. macrotis); } \\
\text { Thong, 2014a [16] (=R. macrotis); } \\
\text { This study }\end{array}$ & $\begin{array}{l}\text { Abramov \& Kruskop } 2012[9](=R . \\
\text { macrotis); Thong } 2008[23] \text { (=R. macrotis); } \\
\text { Thong et al. } 2020[27] \text { (=R. macrotis) }\end{array}$ \\
\hline R. marshalli & Marshall's horseshoe bat & $\begin{array}{l}\text { Thong et al. } 2007 \text { [5]; Thong } 2011 \\
\text { [7]; Abramov \& Kruskop } 2012 \text { [9]; } \\
\text { Thong } 2012 \text { [10]; Kruskop } 2013 \\
\text { [14]; Thong, 2014a [16]; Thong } \\
\text { et al. 2016 [18]; Furey 2002 [20]; } \\
\text { This study }\end{array}$ & $\begin{array}{l}\text { Thong } 2008 \text { [23]; Thong \& Furey } 2008 \\
\text { [24]; Can et al. } 2008 \text { [25]; Thong et al. } \\
\text { 2020 [27]; Thong et al. } 2021 \text { [43] }\end{array}$ \\
\hline R. pearsonii & Pearson's horseshoe bat & $\begin{array}{c}\text { Thong } 2011 \text { [7]; Abramov \& } \\
\text { Kruskop } 2012 \text { [9]; Kruskop } 2013 \\
\text { [14]; Thong, 2014a [16]; Thong } \\
\text { et al. 2016 [18]; Furey } 2002 \text { [20]; } \\
\text { This study }\end{array}$ & $\begin{array}{l}\text { Thong } 2008 \text { [23]; Thong \& Furey } 2008 \\
\text { [24]; Can et al. } 2008 \text { [25]; Thong et al. } \\
2020 \text { [27] ; Thong et al. } 2021 \text { [43] }\end{array}$ \\
\hline R. pusillus & Least horseshoe bat & $\begin{array}{c}\text { Thong } 2011 \text { [7]; Abramov \& } \\
\text { Kruskop } 2012 \text { [9] (= R. pusillus } \\
\text { and R. cf. pusillus); Kruskop } 2013 \\
\text { [14]; Thong, 2014a [16]; Thong } \\
\text { et al. } 2016 \text { [18]; Furey } 2002 \text { [20]; } \\
\text { This study }\end{array}$ & $\begin{array}{l}\text { Thong } 2008 \text { [23]; Thong \& Furey } 2008 \\
\text { [24]; Can et al. } 2008 \text { [25]; Thong et al. } \\
\text { 2020 [27]; Thong et al. } 2021 \text { [43] }\end{array}$ \\
\hline Hipposideridae & Roundleaf bats & & \\
\hline Aselliscus cf. stoliczkanus & Dong Bac trident bat & $\begin{array}{c}\text { Thong } 2011[7] \text { (=A. stoliczkanus); } \\
\text { Abramov \& Kruskop } 2012[9]=A \text {. } \\
\text { stoliczkanus; Kruskop } 2013[14]=A \text {. } \\
\text { stoliczkanus; Thong et al. 2016 [18]; } \\
\text { Furey 2002 [20] (=A. stoliczkanus); } \\
\text { This study }\end{array}$ & $\begin{array}{c}\text { Thong } 2008 \text { [23] =A. stoliczkanus; Thong } \\
\& \text { Furey } 2008 \text { [24] =A. stoliczkanus; Can } \\
\text { et al. } 2008 \text { [25] =A. stoliczkanus; Thong } \\
\text { et al. } 2020 \text { [27] }\end{array}$ \\
\hline Coelops frithii & Tailless leaf-nosed bat & Furey 2002 [20] & $\begin{array}{l}\text { Abramov \& Kruskop } 2012 \text { [9]; Thong } \\
2008 \text { [23]; Thong \& Furey } 2008 \text { [24]; Can } \\
\text { et al. } 2008 \text { [25]; Thong et al. } 2020 \text { [27] }\end{array}$ \\
\hline Hipposideros alongensis & Ha Long leaf-nosed bat & $\begin{array}{c}\text { Bourret 1942a [2] (=H. larvatus } \\
\text { alongensis); Bourret 1942b [3] (=H. } \\
\text { larvatus alongensis); Topal 1993 [4] } \\
\text { (=H. turpis); Thong 2011 [7]; } \\
\text { Abramov \& Kruskop 2012 [9] (=H. } \\
\text { turpis alongensis); Thong et al. } \\
\text { 2012a [11]; Kruskop 2013 [14]; } \\
\text { Thong 2013 [15]; Thong et al. 2016 } \\
\text { [18]; Furey 2002 [20] (=H. turpis); } \\
\text { This study }\end{array}$ & $\begin{array}{l}\text { Thong } 2008 \text { [23] (=H. cf. turpis } \\
\text { alongensis); Thong \& Furey } 2008 \text { [24] } \\
\text { (=H. turpis); Can et al. } 2008 \text { [25] }(=H \text {. } \\
\text { turpis); Thong et al. } 2020 \text { [27] }\end{array}$ \\
\hline
\end{tabular}


Table 4. Cont.

\begin{tabular}{|c|c|c|c|}
\hline Scientific Name & Common Name & $\begin{array}{c}\text { Records with Reference to } \\
\text { Material(s) }\end{array}$ & $\begin{array}{c}\text { Recorded without Reference to } \\
\text { Material(s) }\end{array}$ \\
\hline H. armiger & Great Himalayan leaf-nosed bat & $\begin{array}{c}\text { Thong } 2011 \text { [7]; Abramov \& } \\
\text { Kruskop } 2012 \text { [9]; Thong et al. } \\
\text { 2016 [18]; Furey 2002 [20]; This } \\
\text { study }\end{array}$ & $\begin{array}{c}\text { Kruskop } 2013 \text { [14]; Canh et al. } 1997 \text { [21]; } \\
\text { Thong } 2008 \text { [23]; Thong \& Furey } 2008 \\
\text { [24]; Can et al. } 2008 \text { [25]; Thong et al. } \\
2020 \text { [27] }\end{array}$ \\
\hline H. gentilis & Andersen's roundleaf bat & $\begin{array}{c}\text { Thong } 2011 \text { [7] (=H. pomona); } \\
\text { Abramov \& Kruskop 2012 [9] }(=H . \\
\text { pomona); Thong et al. 2012c [13]; } \\
\text { Kruskop } 2013 \text { [14] (=H. pomona); } \\
\text { Thong et al. 2016 [18] (=H. } \\
\text { pomona); Yuzefovich et al. 2021 } \\
\text { [19]; Furey 2002 [20] (=H. pomona); } \\
\text { This study }\end{array}$ & $\begin{array}{c}\text { Thong } 2008 \text { [23] (=H. pomona); Thong \& } \\
\text { Furey 2008 [24] (=H. pomona); Can et al. } \\
2008 \text { [25] (=H. pomona); Thong et al. } 2020 \\
\text { [27] (=H. pomona). }\end{array}$ \\
\hline H. larvatus & Intermediate leaf-nosed bat & $\begin{array}{l}\text { Bourret 1942b [3]; Thong } 2011 \text { [7] } \\
\text { (=H. grandis); Abramov \& } \\
\text { Kruskop 2012 [9]; Kruskop } 2013 \\
\text { [14]; Thong et al. 2016 [18] (=H. } \\
\text { grandis); Furey 2002 [20]; This } \\
\text { study }\end{array}$ & $\begin{array}{l}\text { Canh et al. } 1997 \text { [21]; Thong } 2008 \text { [23]; } \\
\text { Thong \& Furey } 2008 \text { [24]; Can et al. } 2008 \\
\text { [25]; Thong et al. } 2020 \text { [27] (=H. grandis). }\end{array}$ \\
\hline H. griffini & Griffin's leaf-nosed bat & $\begin{array}{c}\text { Thong } 2011 \text { [7]; Thong et al. 2012b } \\
\text { [12] }\end{array}$ & $\begin{array}{l}\text { Abramov \& Kruskop } 2012 \text { [9]; Thong } \\
2008 \text { [23] (=Hipposideros sp.nov.); } \\
\text { Kruskop 2013 [14]; Thong et al. 2020 [27] }\end{array}$ \\
\hline H. khaokhouayensis & Phou Khao Khouay leaf-nosed bat & $\begin{array}{l}\text { Thong et al. } 2008 \text { [6]; Thong } 2011 \\
\text { [7]; Abramov \& Kruskop } 2012 \text { [9]; } \\
\text { Kruskop } 2013 \text { [14]; Thong et al. } \\
2016 \text { [18]; This study }\end{array}$ & $\begin{array}{c}\text { Thong 2008 [23]; Thong \& Furey } 2008 \\
\text { [24]; Thong et al. } 2020 \text { [27] }\end{array}$ \\
\hline Vespertilionidae & Common bats & & \\
\hline Harpiocephalus harpia & Lesser hairy-winged bat & Furey 2002 [20]; & $\begin{array}{l}\text { Abramov \& Kruskop } 2012 \text { [9]; Thong } \\
2008 \text { [23]; Thong \& Furey } 2008 \text { [24]; Can } \\
\text { et al. 2008 [25]; Thong et al. 2020 [27] }\end{array}$ \\
\hline Hypsugo pulveratus & Chinese pipistrelle & Thong et al. 2016 [18]; This study & $\begin{array}{c}\text { Abramov \& Kruskop } 2012 \text { [9]; Thong } \\
2008 \text { [23]; Thong \& Furey 2008 [24]; } \\
\text { Thong et al. } 2020 \text { [27] }\end{array}$ \\
\hline Murina cyclotis & Round-eared tube-nosed bat & Furey 2002 [20]; This study & $\begin{array}{c}\text { Abramov \& Kruskop } 2012 \text { [9]; Kruskop } \\
2013 \text { [14]; Thong } 2008 \text { [23]; Thong \& } \\
\text { Furey 2008 [24]; Can et al. 2008 [25]; } \\
\text { Thong et al. } 2020 \text { [27] }\end{array}$ \\
\hline M. harrisoni & Harrison's murine bat & Thong et al. 2011 [8] & $\begin{array}{c}\text { Abramov \& Kruskop 2012 [9] } \\
\text { (=M.tiensa); Kruskop 2013 [14]; Thong } \\
2008 \text { [23] (=M. tiensa); Thong et al. } 2020 \\
\text { [27] }\end{array}$ \\
\hline Myotis alticraniatus & Small-toothed myotis & $\begin{array}{l}\text { Abramov \& Kruskop } 2012 \text { [9] (=M. } \\
\text { siligorensis); Kruskop } 2013 \text { [14] } \\
\text { (=M. siligorensis); Thong et al. } 2016 \\
\text { [18] (=M. siligorensis); This study }\end{array}$ & $\begin{array}{l}\text { Thong } 2008 \text { [23] (=M. siligorensis); Thong } \\
\quad \text { \& Furey } 2008 \text { [24] (=M. siligorensis); } \\
\text { Thong et al. } 2020 \text { [27] (=M. siligorensis) }\end{array}$ \\
\hline M. hasseltii & Lesser large-footed myotis & This study & \\
\hline M. muricola & Nepalese whiskered bat & Furey 2002 [20]; & $\begin{array}{l}\text { Abramov \& Kruskop } 2012 \text { [9]; Thong } \\
2008 \text { [23]; Thong \& Furey } 2008 \text { [24]; Can } \\
\text { et al. 2008 [25]; Thong et al. } 2020 \text { [27] }\end{array}$ \\
\hline M. pilosus & Rickett's big-footed myotis & $\begin{array}{c}\text { Thong et al. } 2016 \text { (=Myotis sp.) } \\
\text { [18]; This study }\end{array}$ & Thong et al. 2020 [27] (=M. cf. pilosus) \\
\hline Phoniscus jagorii & Peters' trumpet-eared bat & This study & \\
\hline Pipistrellus abramus & Japanese Pipistrelle & This study & $\begin{array}{c}\text { Abramov \& Kruskop } 2012 \text { [9]; Kruskop } \\
\text { 2013 [14]; Borrisenko\&Kruskop } 2003 \\
\text { [22]; Thong } 2008 \text { [23]; Thong \& Furey } \\
\text { 2008 [24]; Can et al. 2008 [25]; Thong } \\
\text { et al. } 2020 \text { [27] }\end{array}$ \\
\hline
\end{tabular}


Table 4. Cont.

\begin{tabular}{|c|c|c|c|}
\hline Scientific Name & Common Name & $\begin{array}{c}\text { Records with Reference to } \\
\text { Material(s) }\end{array}$ & $\begin{array}{c}\text { Recorded without Reference to } \\
\text { Material(s) }\end{array}$ \\
\hline P. javanicus & Javan pipistrelle & Furey 2002 [20] & $\begin{array}{l}\text { Abramov \& Kruskop } 2012 \text { [9]; Kruskop } \\
2013 \text { [14]; Canh et al. } 1997 \text { [21]; Thong } \\
2008 \text { [23]; Thong \& Furey 2008 [24]; Can } \\
\text { et al. 2008 [25]; Thong et al. } 2020 \text { [27] }\end{array}$ \\
\hline P. tenuis & Least pipistrelle & $\begin{array}{c}\text { Thong et al. } 2016 \text { [18]; Furey } 2002 \\
\text { [20] }\end{array}$ & $\begin{array}{l}\text { Abramov \& Kruskop } 2012 \text { [9]; Thong } \\
2008 \text { [23]; Thong \& Furey } 2008 \text { [24]; Can } \\
\text { et al. 2008 [25]; Thong et al. } 2020 \text { [27] }\end{array}$ \\
\hline Tylonycteris fulvida & Small bamboo bat & This study & \\
\hline Miniopteridae & Bent-wing bats & & \\
\hline Miniopterus fuliginosus & Eastern bent-winged bat & $\begin{array}{c}\text { Thong et al. } 2016[18] \\
\text { (=Miniopterus cf. magnater); This } \\
\text { study }\end{array}$ & $\begin{array}{c}\text { Abramov \& Kruskop 2012 [9] } \\
\text { (=Miniopterus cf. fuliginosus); Kruskop } \\
2013 \text { [14]; Thong 2008 [23] =Miniopterus } \\
\text { cf. fuliginosus); Thong \& Furey 2008 [24] } \\
\text { (=Miniopterus sp.); Nga \& Tung 2018 } \\
\text { [26]; Thong et al. } 2020 \text { [27] }\end{array}$ \\
\hline
\end{tabular}

\subsection{Species Recorded over the Surveys}

4.1.1. Cynopterus sphinx

One male and one female were recorded from mangrove in the Cat Ba Island (Tables 1 and 2; Figures 1 and 2). Their morphological features were similar to previous descriptions $[14,44,45]$. Its occurrence in CBBR was mentioned in seven previous publications (Table 4). Canh et al. included the first record of this species without reference to any material [21]. Furey included a record of this species with reference to a specimen collected over a field survey between 20 July and 30 August 1999 [20]. This record was cited in four subsequent publications [23-25,27]. This species is uncommon in CBBR [27]. It was observed while it was flying over a road near the Headquarters of Cat Ba National Park [9]. This is the first record of Cynopterus sphinx from the mangrove ecosystem.

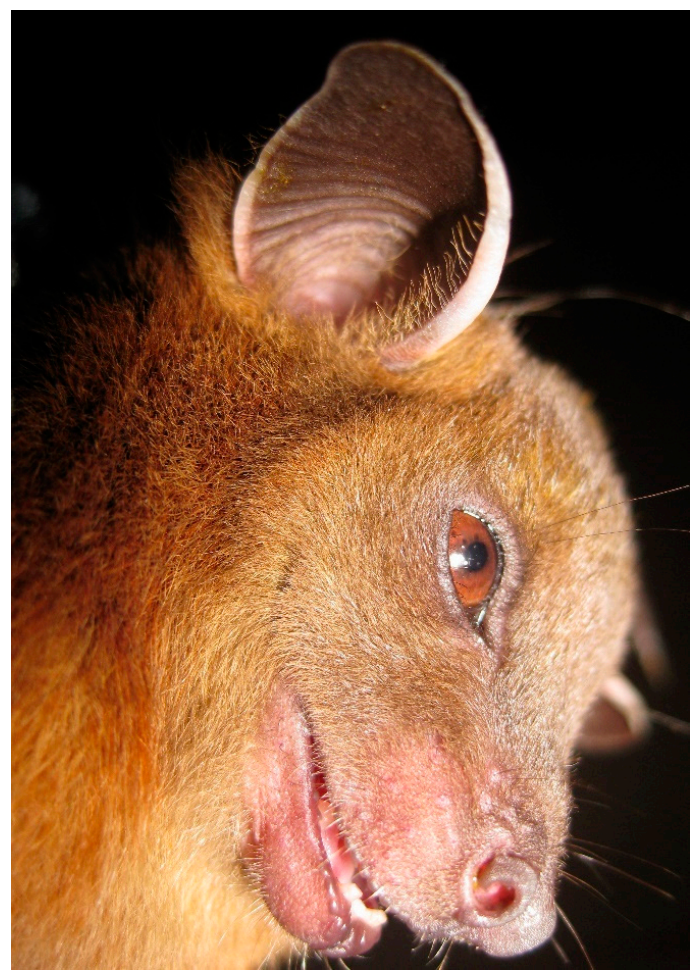

Figure 2. Cynopterus sphinx, adult male. Photograph by Vu Dinh Thong. 


\subsubsection{Macroglossus minimus}

One male and one female were recorded from mangroves in the Cat Ba Island (Tables 1 and 2; Figures 1 and 3). Their morphological measurements other characteristics are similar to previous descriptions (Tables 2 and 4). This is the first record for CBBR and northern Vietnam. Before the present study, this species was also recorded from mangroves in south-central Vietnam [46]. However, its distribution in Vietnam was only confirmed in south-central and southern Vietnam [14,46,47].

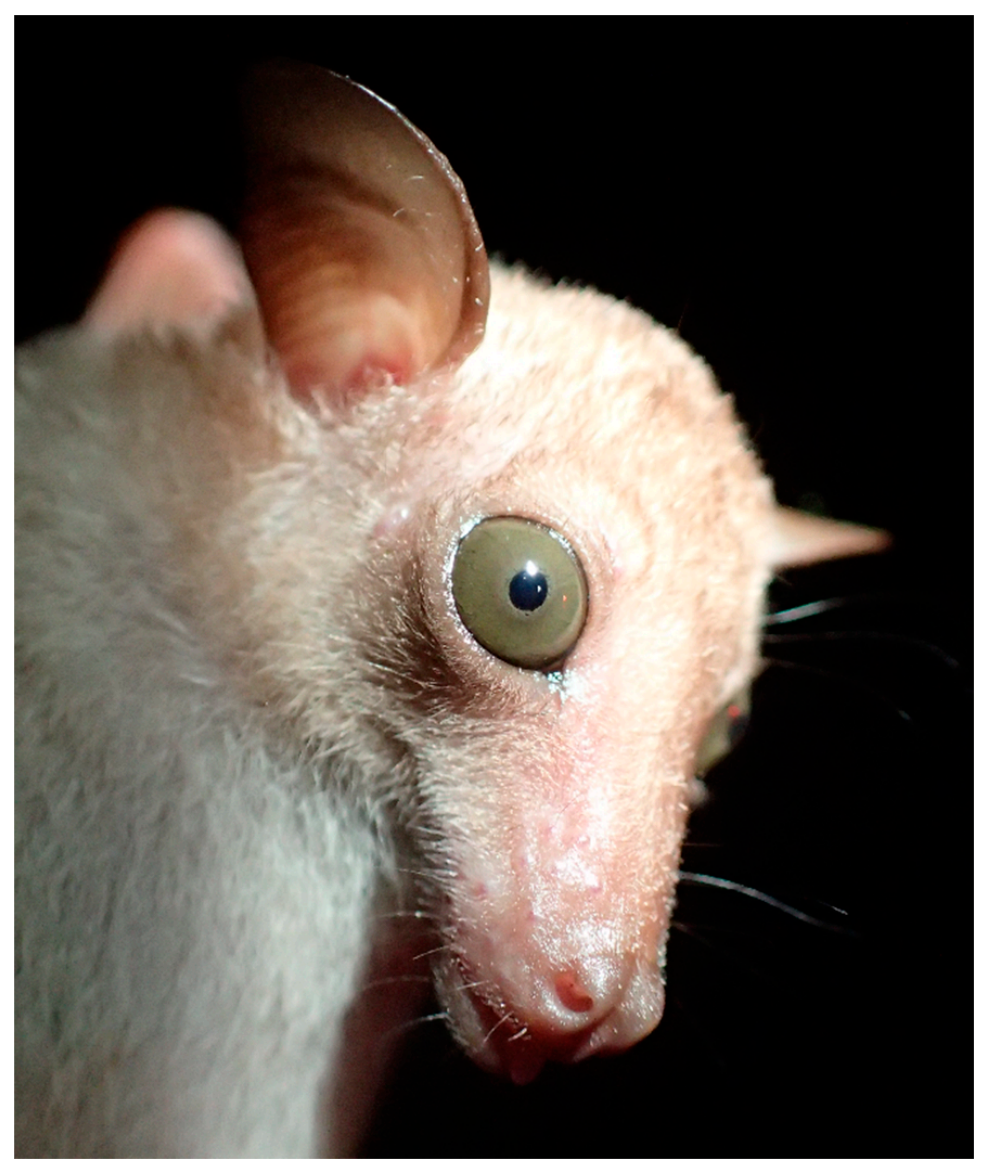

Figure 3. Macroglossus minimus, adult female. Photograph by Vu Dinh Thong.

\subsubsection{Taphozous melanopogon}

Six males and three females were recorded from different habitats at four study sites (Tables 1 and 2; Figures 1 and 4). They were all adults and found in different caves and mangrove areas in Cat Ba Island. Morphological features of captured bats are identical to descriptions in previous publications $[14,45,46]$. This species was included in seven previous publications (Table 4). However, Thong et al. were the first to document records with reference to primary materials of this species from the Cat Ba Island [18]. Between 2015 and 2020, this species was commonly detected by acoustic recordings at almost all study sites. It emits long multiharmonic calls. The iFM, tFM, and pulse duration values of the second harmonic of two captured bats are in a range of $27.7-28.2 \mathrm{kHz}, 20.3-20.8 \mathrm{kHz}$, and 14.6-14.7 ms, respectively. It was observed in different caves, mangrove areas, and other habitats associated with wetlands in CBBR. 


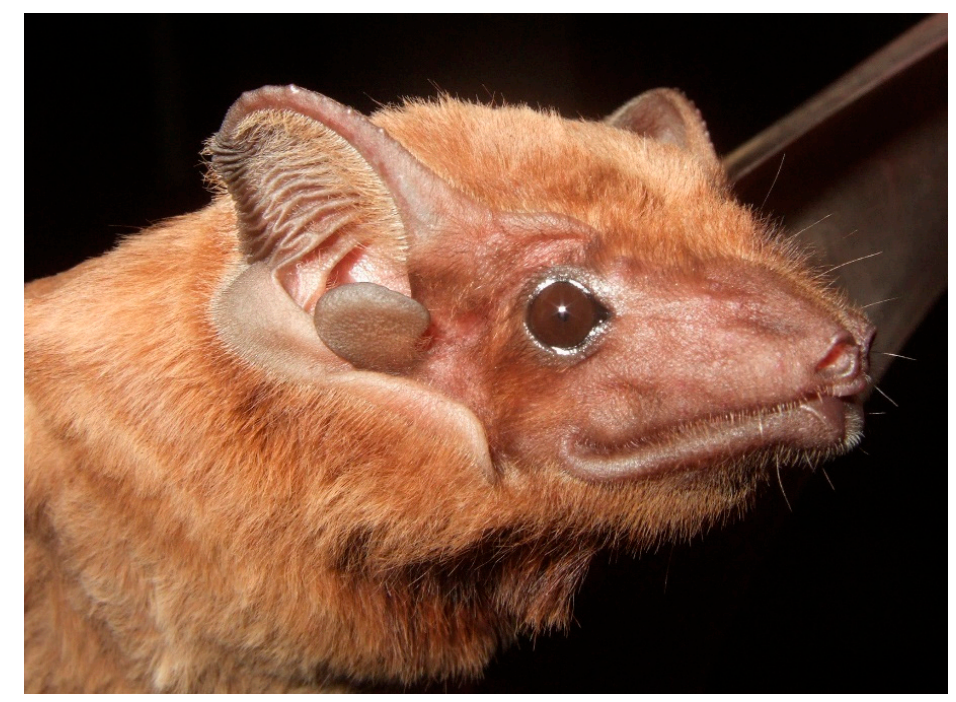

Figure 4. Taphozous melanopogon, adult male. Photograph by Vu Dinh Thong.

\subsubsection{Rhinolophus affinis}

Two males and four females were captured in mangroves in the Cat Ba Island and secondary forests in an unnamed island within CBBR (Tables 1 and 2; Figure 5). Morphological features of the six captured bats are similar to previous descriptions $[7,14,16,36,44,45]$. Previous records of this species from the Cat Ba Island were included in six publications (Table 4). However, only two of these publications referred to materials $[7,16]$. This species is distinguishable from other Rhinolophus species from CBBR in both morphology and echolocation $[7,16]$. The CF2 and pulse duration values of six captured bats are in a range of $72.3-73.6 \mathrm{kHz}$ and $20.7-29.8 \mathrm{~ms}$, respectively.

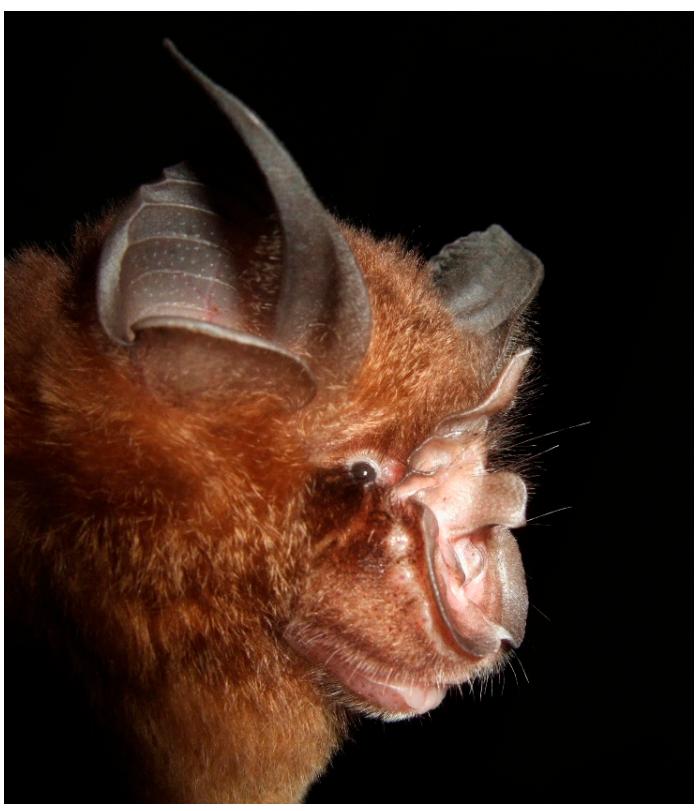

Figure 5. Rhinolophus affinis, adult male. Photograph by Vu Dinh Thong.

\subsubsection{Rhinolophus siamensis}

Three males and three females were captured in mangroves in the Cat Ba Island and secondary forests on an unnamed island (Tables 1 and 2; Figure 6). Morphological features of the captured bats are similar to previous descriptions $[7,14,16,36,44,45]$. Records of this species from CBBR were included in five previous publications as Rhinolophus macrotis 
(Table 4). However, it can be distinguished from Rhinolophus macrotis and other Rhinolophus species from CBBR based on morphological and echolocation features $[7,16,44,45]$. This is the first record of Rhinolophus siamensis from the mangrove ecosystem in Vietnam. The CF2 and pulse duration values of six captured bats are in a range of $75.6-75.8 \mathrm{kHz}$ and 18.4-19.3 ms, respectively.

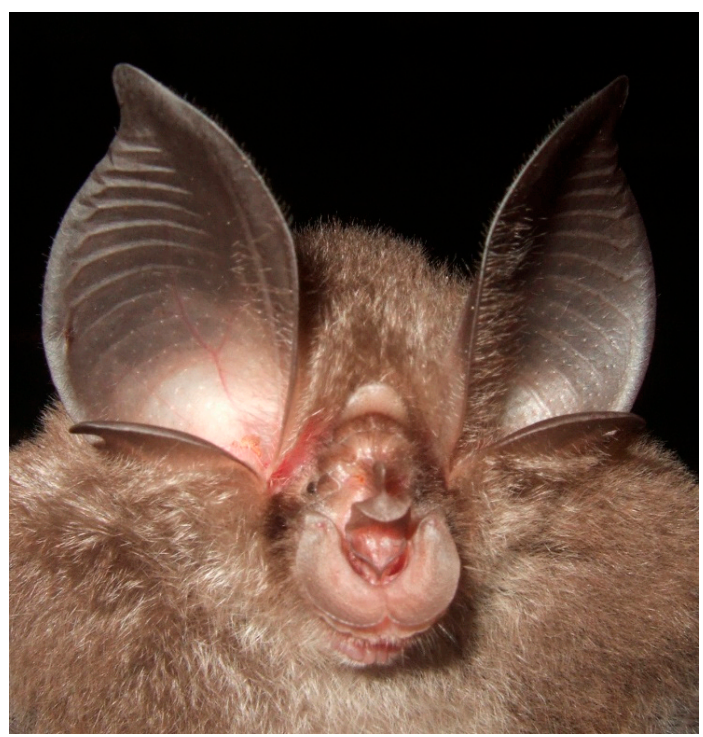

Figure 6. Rhinolophus siamensis, adult male. Photograph by Vu Dinh Thong.

\subsubsection{Rhinolophus marshalli}

Five males and seven females were recorded from mangroves and forests in Cat Ba Island and an unnamed island (Tables 1-3; Figure 7). Morphological features of the captured bats are identical to previous descriptions [7,14,16,36,44,45,48]. Morphology and echolocation of this species from the Cat Ba Island were described in eight previous publications $[5,7,9,10,14,16,18]$. Records of this species were included in at least twelve previous publications (Table 4 ). This species is common in almost all surveyed caves and other habitats in Cat Ba Island but is rare throughout Vietnam. The CF2 and pulse duration values of twelve captured bats are in a range of $43.5-45.6 \mathrm{kHz}$ and $47.9-48.4 \mathrm{~ms}$, respectively.

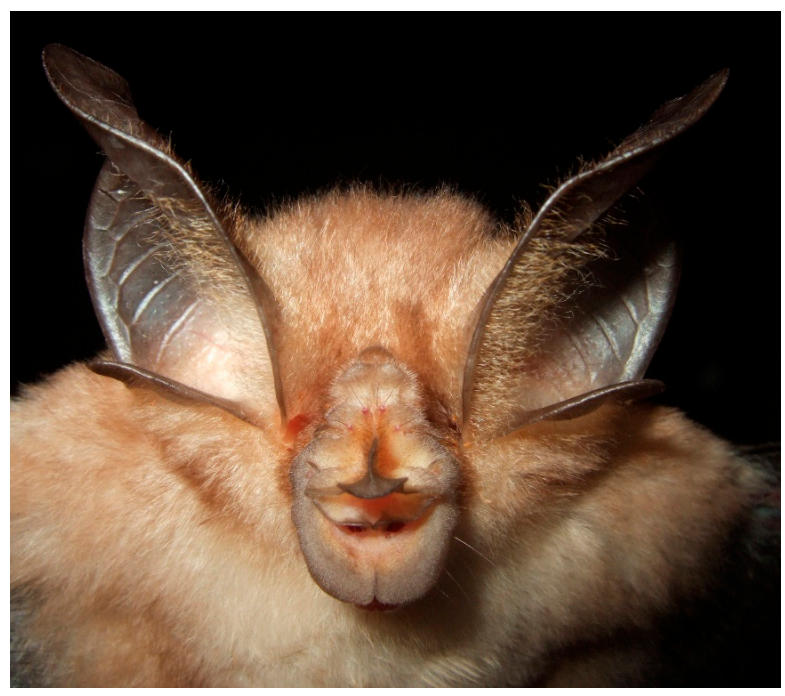

Figure 7. Rhinolophus marshalli, adult male. Photograph by Vu Dinh Thong. 


\subsubsection{Rhinolophus pearsonii}

Four adult males and five adult females were captured in mangroves and forests in the Cat Ba Island (Table 1; Figure 8). Morphological features of the nine captured bats are identical to previous descriptions $[7,14,16,36,44,45]$. The occurrences of this species in forests and caves in Cat Ba Island were included in ten previous publications (Table 4). This species is common in different caves and other habitats in CBBR. The CF2 and pulse duration values of nine captured bats are in a range of $53.6-60.9 \mathrm{kHz}$ and $30.5-37.3 \mathrm{~ms}$, respectively.

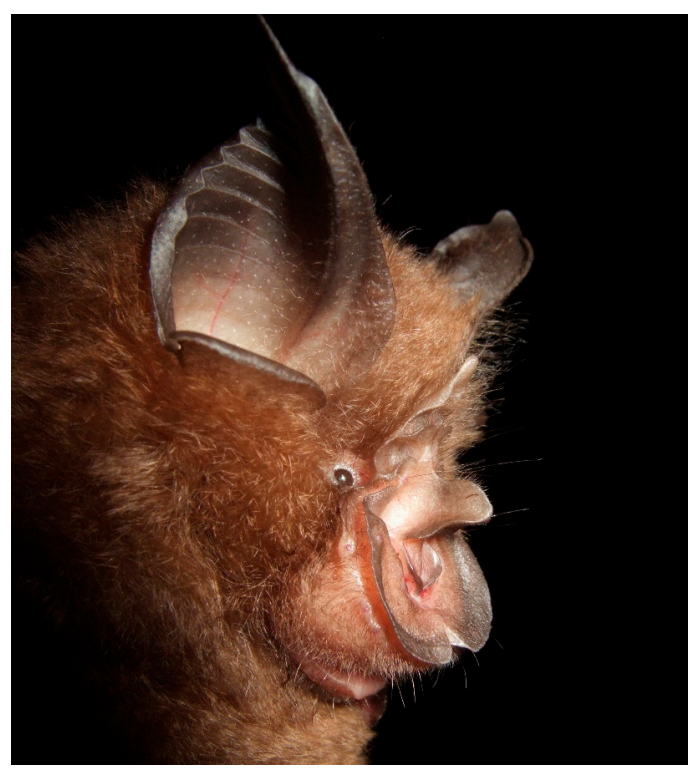

Figure 8. Rhinolophus pearsonii, adult male. Photograph by Vu Dinh Thong.

\subsubsection{Rhinolophus pusillus}

Two adult males and two adult females were captured in mangroves and forests in Cat Ba Island and an unnamed island (Table 1; Figure 9). The taxonomic status of this species is still complicated throughout its distributional range worldwide. Records of this species were included in 11 publications (Table 4). The connecting process of this species in the Cat Ba Island is widely variable, ranging from triangular to pointed hornlike $[9,16]$. Morphological characteristics of the four captured individuals over the surveys exhibit both forms. Abramov and Kruskop (2012) provisionally regarded the "triangular" and "pointed horn-like" forms as R. cf. pusillus and R. cf. subbadius, respectively [9]. However, these both forms were subsequently identified as $R$. pusillus $[14,16,18,48]$. The CF2 and pulse duration values of four captured bats are in a range of $101.3-113.6 \mathrm{kHz}$ and $39.6-41.4 \mathrm{~ms}$, respectively.

\subsubsection{Aselliscus cf. stoliczkanus}

One male and seven females were captured in caves and forests in Cat Ba Island (Tables 1-3; Figure 10). This species form was detected and observed in almost all caves surveyed in Cat Ba Island between 2015 and 2020. Previous records of this species in CBBR were included in previous publications (Table 4). With long canines and freshy posterior nose-leaf, the eight captured bats from CBBR are morphologically similar to $A$. dongbacanus [44,45]. The taxonomic status of this species form will be confirmed according to data on both morphology and genetics in the future. The CF2 and pulse duration values of six captured bats are in a range of $123.5-123.8 \mathrm{kHz}$ and $4.0-6.2 \mathrm{~ms}$, respectively. 


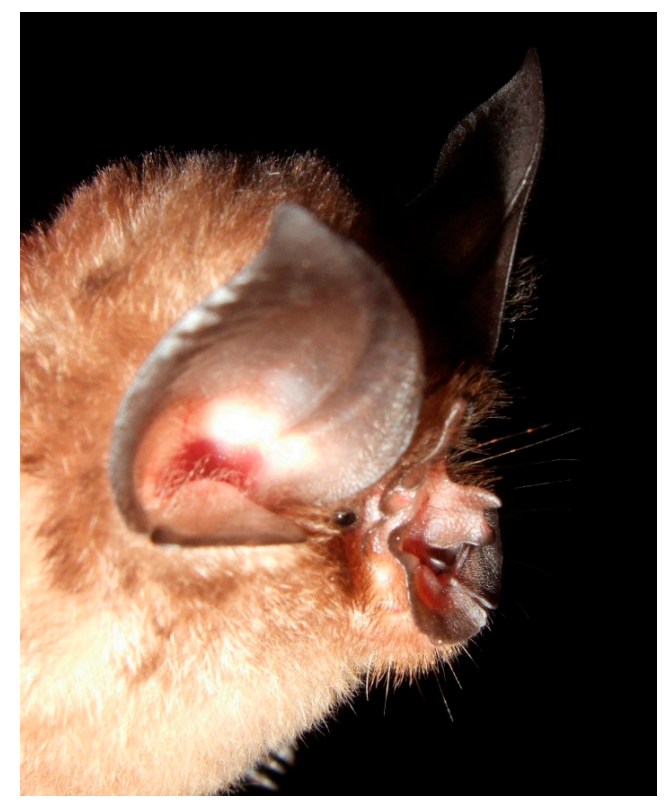

Figure 9. Rhinolophus pusillus, adult male. Photograph by Vu Dinh Thong.

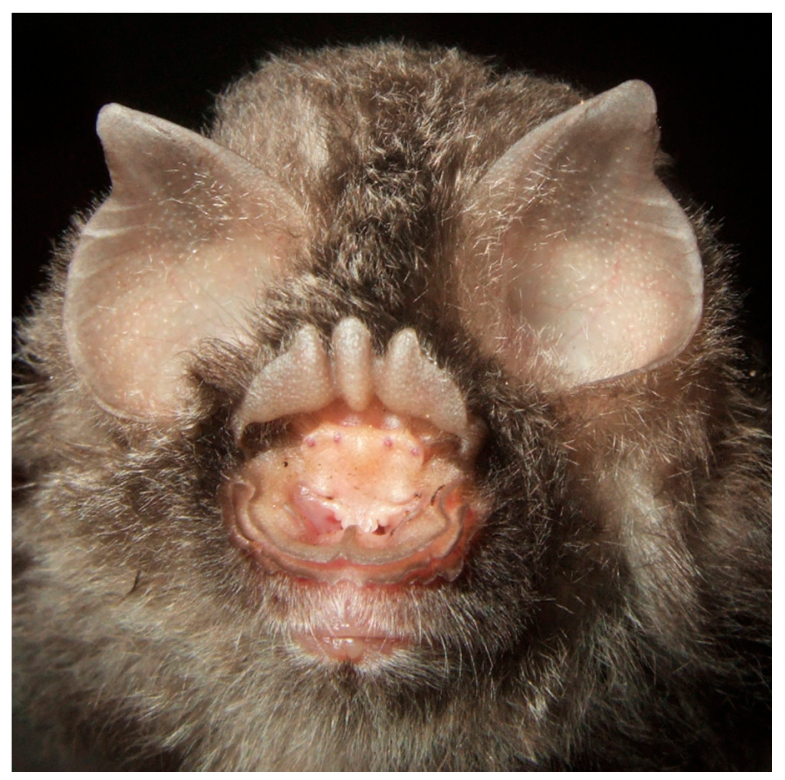

Figure 10. Aselliscus cf. stoliczkanus, adult female. Photograph by Vu Dinh Thong.

\subsubsection{Hipposideros alongensis}

Two adult females were captured in two caves in the Cat Ba Island (Tables 1 and 2; Figure 11). This species was first recorded from the Cat Ba Island as Hipposideros larvatus alongensis [2,3]. It was subsequently regarded as Hipposideros turpis alongensis [4,7]. Since 2012, its nomenclature has been confirmed as Hipposideros alongensis, which is endemic to Vietnam and comprises two subspecies: H. a. alongensis from the Ha Long Bay area including CBBR and H. a. sungi from the mainland area in northeastern Vietnam [11]. To date, records of this species from CBBR were included in 14 previous publications (Table 4). It was commonly observed and detected by echolocation recorders in almost all caves and other habitats in Cat Ba Island. The CF2 and pulse duration values of two captured bats are in a range of $77.0-79.6 \mathrm{kHz}$ and $6.2-6.8 \mathrm{~ms}$, respectively. 


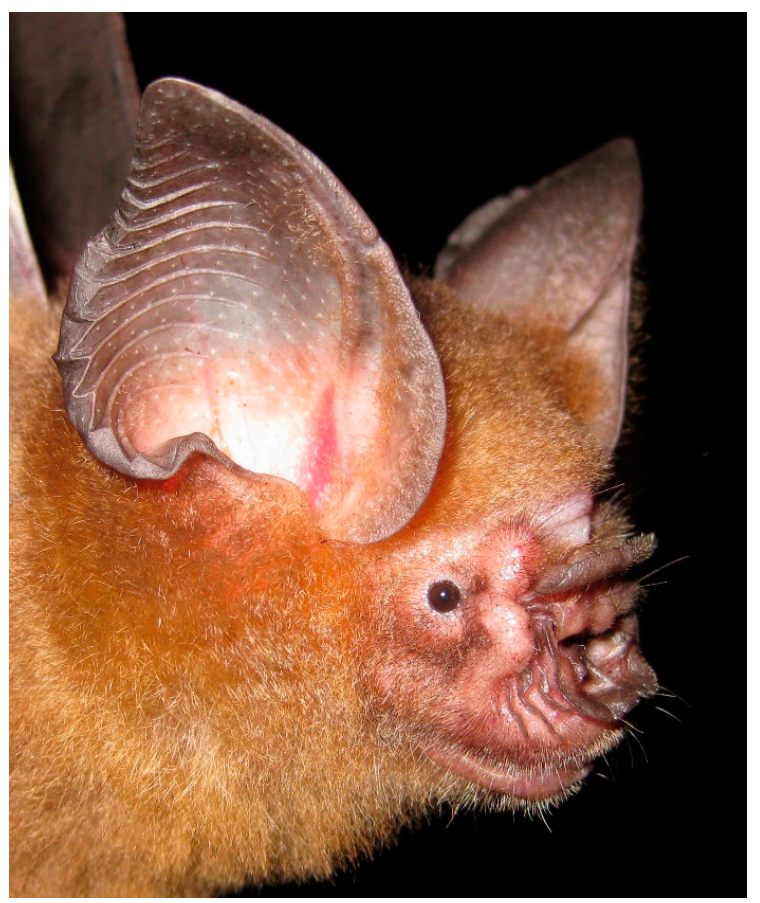

Figure 11. Hipposideros alongensis, adult male. Photograph by Vu Dinh Thong.

\subsubsection{Hipposideros armiger}

Two adult males and one adult female were captured in caves and forests in Cat Ba Island (Tables 1 and 2; Figure 12). Morphological features of the captured bats are identical to previous descriptions $[7,14,44,45]$. This species and $H$. griffini are similar in external morphology but distinguishable in all craniodental, acoustic, and genetic aspects $[12,45,49]$. Its occurrence in CBBR was included in ten previous publications (Table 4). Over the surveys, it was commonly observed and detected by echolocation recorders in different caves in Cat Ba Island. The CF2 and pulse duration values of three captured bats are in a range of $64.5-68.8 \mathrm{kHz}$ and $8.9-11.9 \mathrm{~ms}$, respectively.

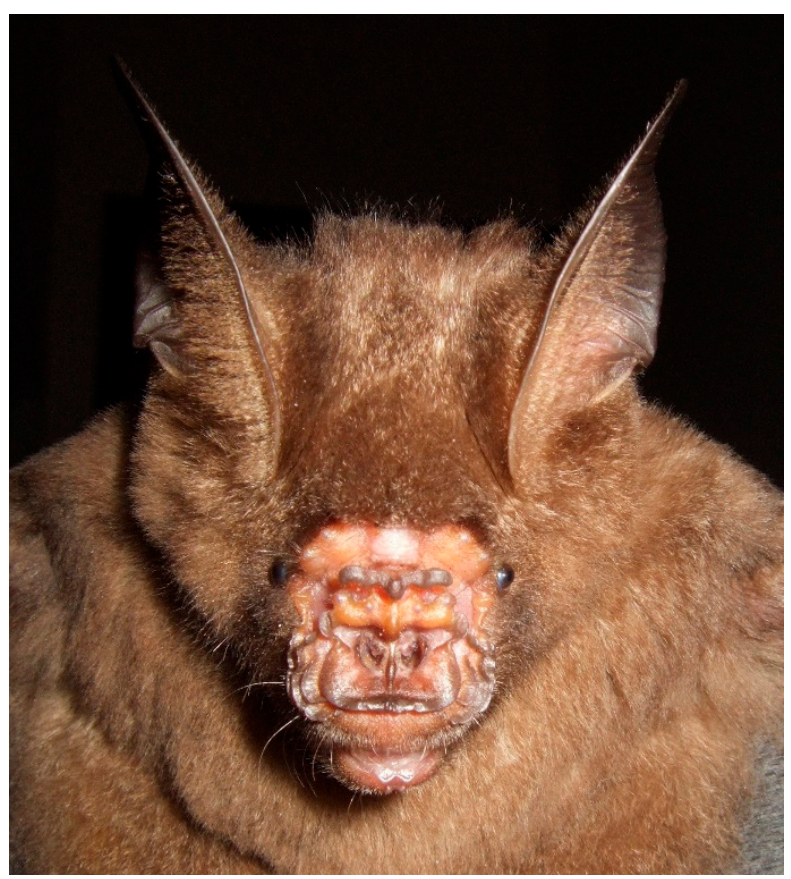

Figure 12. Hipposideros armiger, adult male. Photograph by Vu Dinh Thong. 


\subsubsection{Hipposideros gentilis}

Three adult males and seven adult females were captured in mangroves and forests in Cat Ba Island (Tables 1-3; Figure 13). Previous records of this species from CBBR were regarded as Hipposideros pomona in ten previous publications (Table 4). Morphological features of the ten captured bats are similar to previous descriptions of "Hipposideros pomona" $[14,44,45]$. The taxonomic status of Hipposideros gentilis from CBBR and several other areas in Vietnam has been confirmed by Yuzefovich et al. [19]. This species is commonly observed in almost all caves in Cat Ba Island. Before the present study, it was only recorded from caves and forests. This is the first record of Hipposideros gentilis from mangrove in Vietnam. The CF2 and pulse duration values of six captured bats are in a range of $119.1-123.1 \mathrm{kHz}$ and $6.2-6.9 \mathrm{~ms}$, respectively.

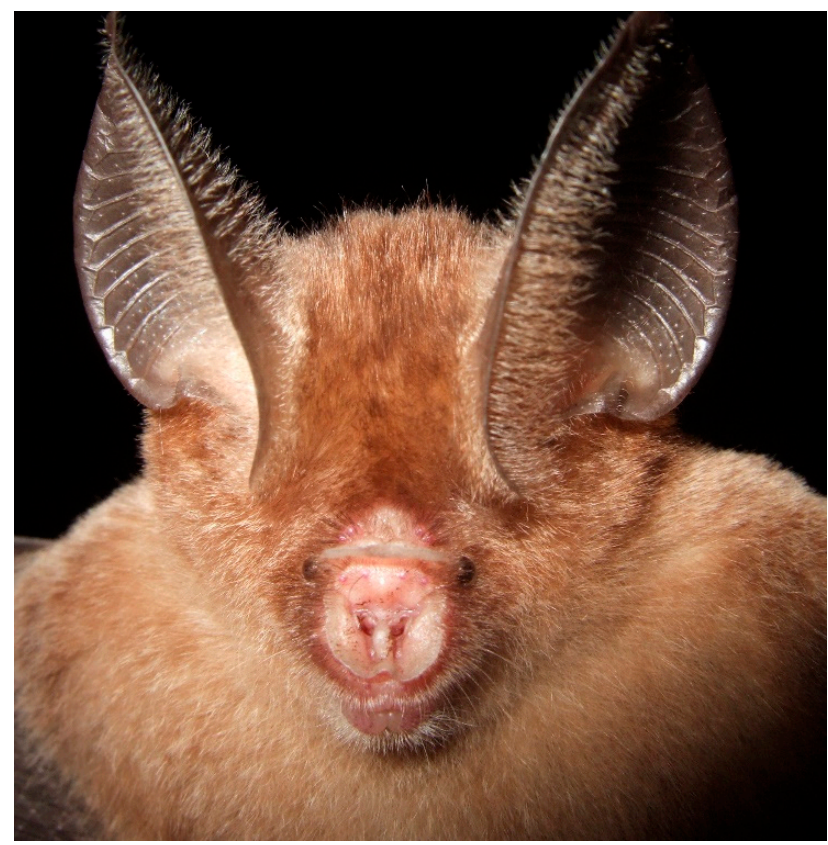

Figure 13. Hipposideros gentilis, adult male. Photograph by Vu Dinh Thong.

\subsubsection{Hipposideros larvatus}

Seven males and twelve females were captured in mangroves, caves, and forests in the Cat Ba Island and two unnamed islands (Tables 1-3; Figure 14). Morphological features of the captured bats are identical to previous descriptions $[7,9,14,44,45]$. This is one of the most common bat species in the Cat Ba Island with records in different caves and other habitats. Previous records of this species from CBBR were included in 11 publications including the records regarded as Hipposideros grandis (Table 4).

The taxonomic status of this species complex is still under discussion. Morphological characteristics and echolocation frequencies of this species are in a wide variation throughout its distributional range $[7,9,14,45]$. Echolocation frequencies of this species in CBBR are similar to those of $H$. grandis in southern Vietnam $[7,9,18]$. However, they are identical to the 'typical' Hipposideros larvatus in morphology. Further study is required to resolve the taxonomic status of this species and its whole distributional range. The CF2 and pulse duration values of six captured bats are in a range of $95.6-102.6 \mathrm{kHz}$ and 3.9-6.9 ms, respectively.

\subsubsection{Hipposideros khaokhouayensis}

One male and one female were captured in mangrove and forest in Cat Ba Island (Tables 1-3; Figure 15). This "vulnerable" species is quite common, with previous records in almost all surveyed caves in the Cat Ba Island $[6,7,9,14,18]$. Its occurrence in CBBR was included in eight publications (Table 4). It is distinguishable from all remaining Hipposideros 
species in CBBR by the disc-like internarial septum $[6,7,9,14]$. To date, this species is only known from a small area in central Laos and the Cat Ba Island $[6,7,9,14,50]$. This is the first record from a mangrove ecosystem. The CF2 and pulse duration values of two captured bats are in a range of $91.2-92.1 \mathrm{kHz}$ and $7.7-8.8 \mathrm{~ms}$, respectively.

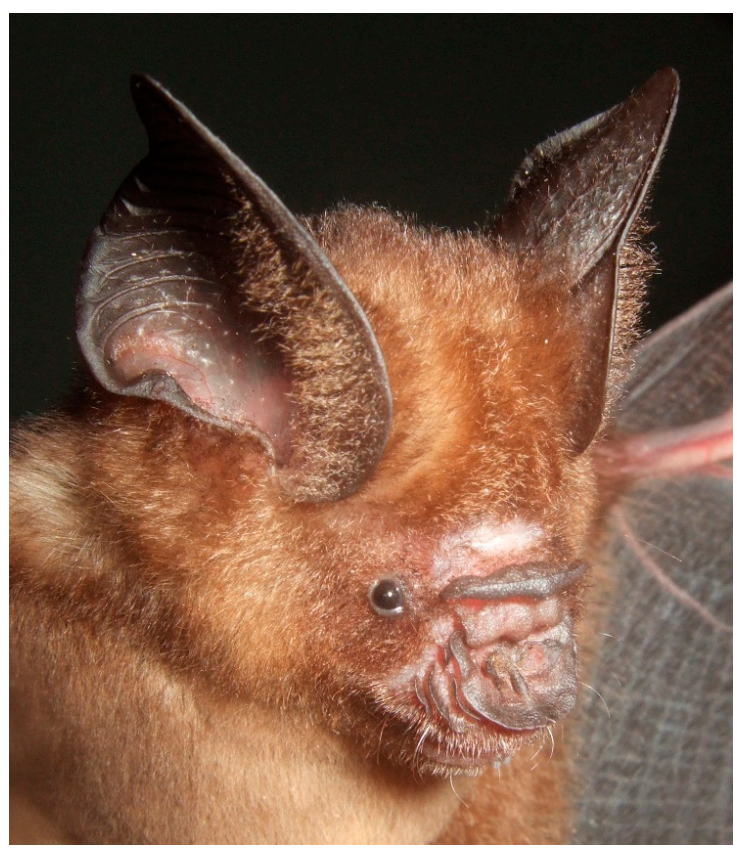

Figure 14. Hipposideros larvatus, adult male. Photograph by Vu Dinh Thong.

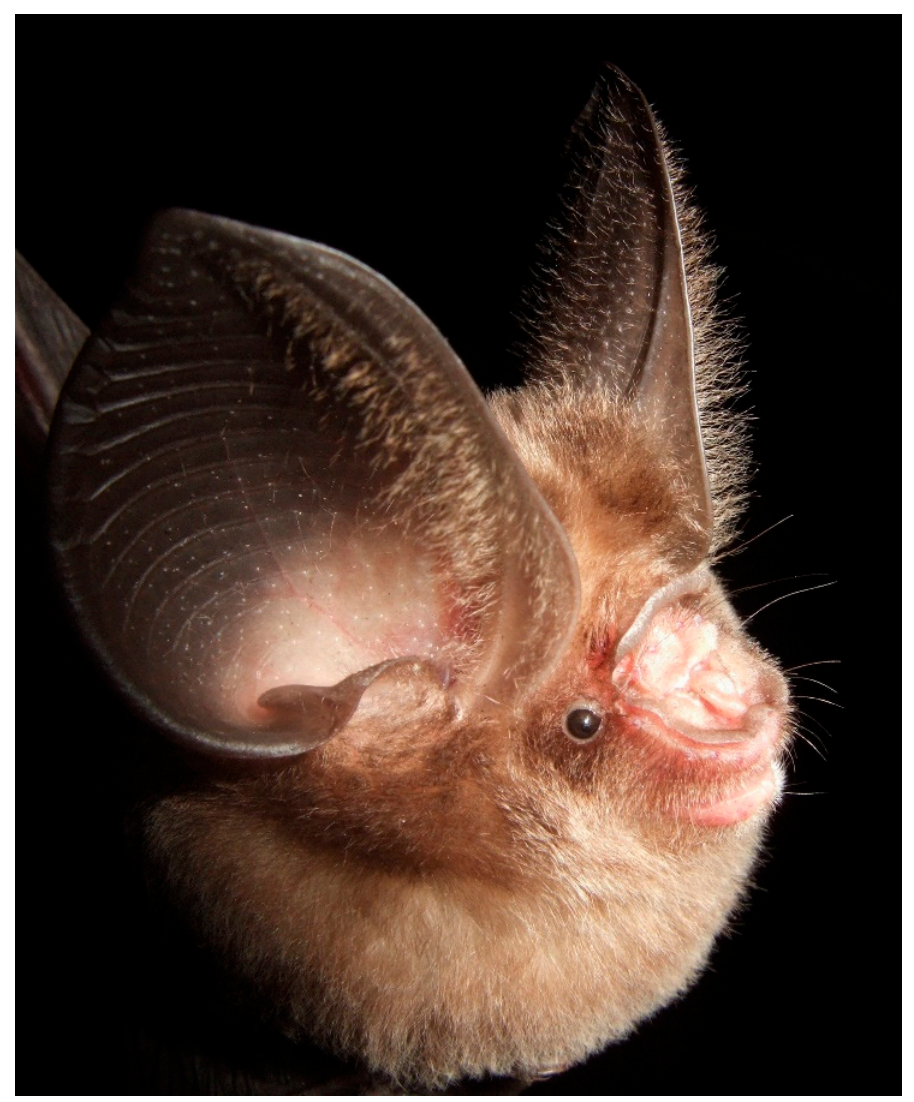

Figure 15. Hipposideros khaokhouayensis, adult male. Photograph by Vu Dinh Thong. 


\subsubsection{Hypsugo pulveratus}

Six males and seven females were captured in mangroves, caves, and forests in Cat $\mathrm{Ba}$ Island and three other unnamed islands (Tables 1 and 2; Figure 16). The morphological features of this species are identical to previous descriptions [14,44,45]. Records of this species were included in five previous publications (Table 4). It was commonly recorded in different caves and other habitats in the Cat Ba Island and other unnamed islands in CBBR. This is the first record of Hypsugo pulveratus from a mangrove in Vietnam. The $\mathrm{iFM}, \mathrm{tFM}$, and pulse duration values of five captured bats are in a range of $82.7-89.2 \mathrm{kHz}$, $38.5-39.0 \mathrm{kHz}$, and $1.8-2.6 \mathrm{~ms}$, respectively.

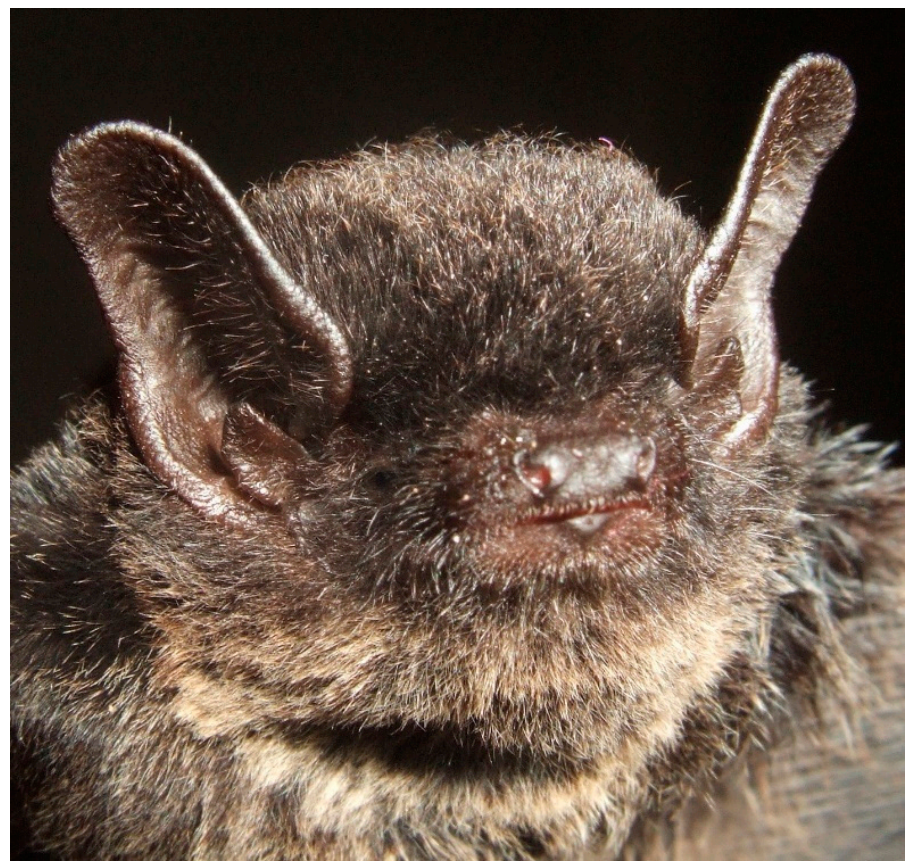

Figure 16. Hypsugo pulveratus, adult male. Photograph by Vu Dinh Thong.

\subsubsection{Murina cyclotis}

Two females were captured in forests in the Cat Ba Island and an unnamed island in CBBR (Tables 1 and 2; Figure 17). Morphological features of the two captured bats are similar to previous descriptions $[14,44,45]$. Before the present study, the only specimen of this species from CBBR was reported by Furey (2002) [20], which was cited in six subsequent publications (Table 4). The iFM, tFM, and pulse duration values of two captured bats are in a range of $163.4-167.7 \mathrm{kHz}, 55.9-56.8 \mathrm{kHz}$, and $2.8-4.3 \mathrm{~ms}$, respectively.

\subsubsection{Myotis hasseltii}

One male and one female were captured in mangroves in Cat Ba Island (Tables 1 and 2; Figure 18). Their morphological features are similar to previous descriptions [44,45]. Identification of the two captured bats was based on morphological and molecular aspects (Figure 19). This species was regarded as an uncommon species, with rare records from Vietnam [51]. However, it was commonly detected by acoustic recordings and observed in mangroves in the Cat Ba Island and other mangrove areas in Vietnam. The iFM, tFM, and pulse duration values of two captured bats are in a range of $86.4-92.1 \mathrm{kHz}, 46.5-47.8 \mathrm{kHz}$, 2.2-2.7 ms, respectively. 


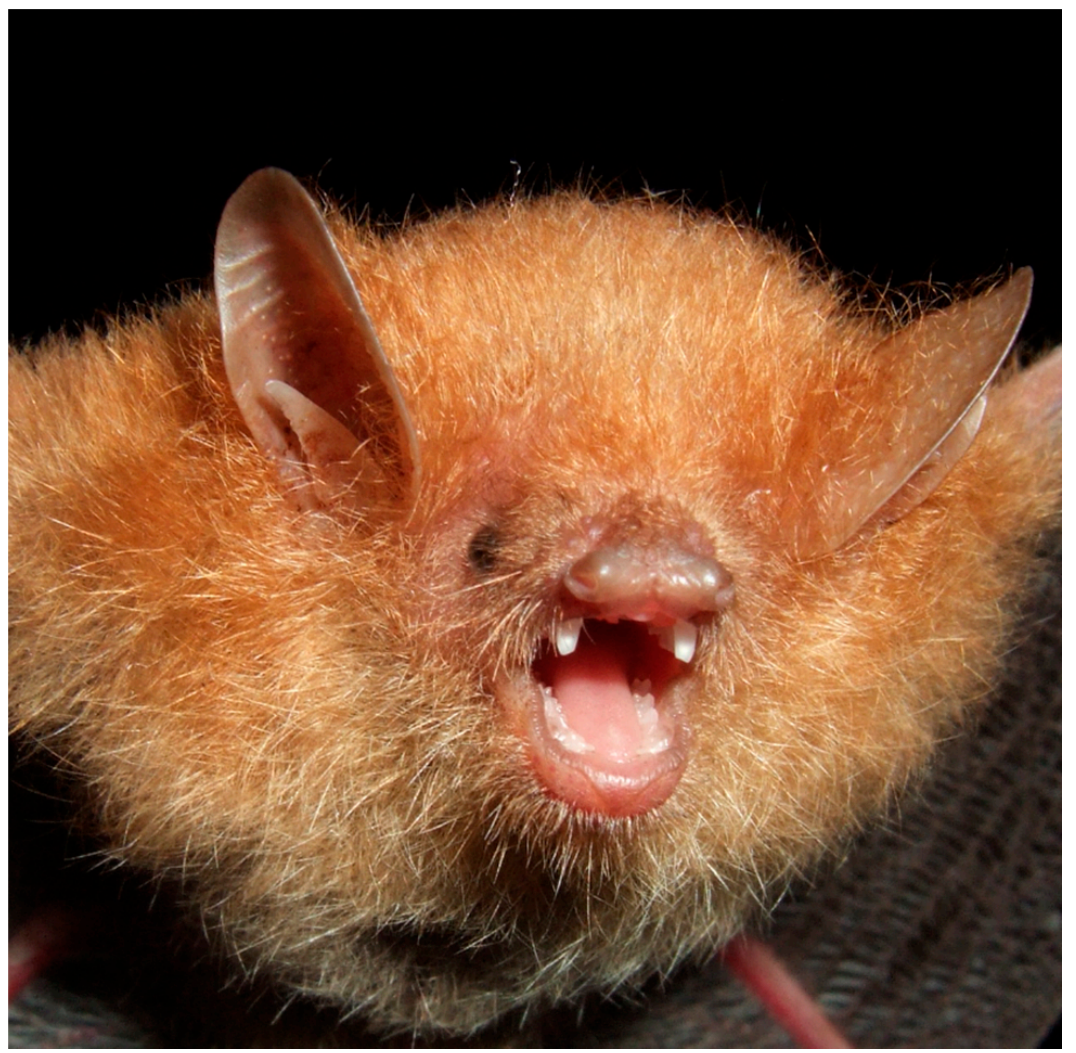

Figure 17. Murina cyclotis, adult male. Photograph by Vu Dinh Thong.

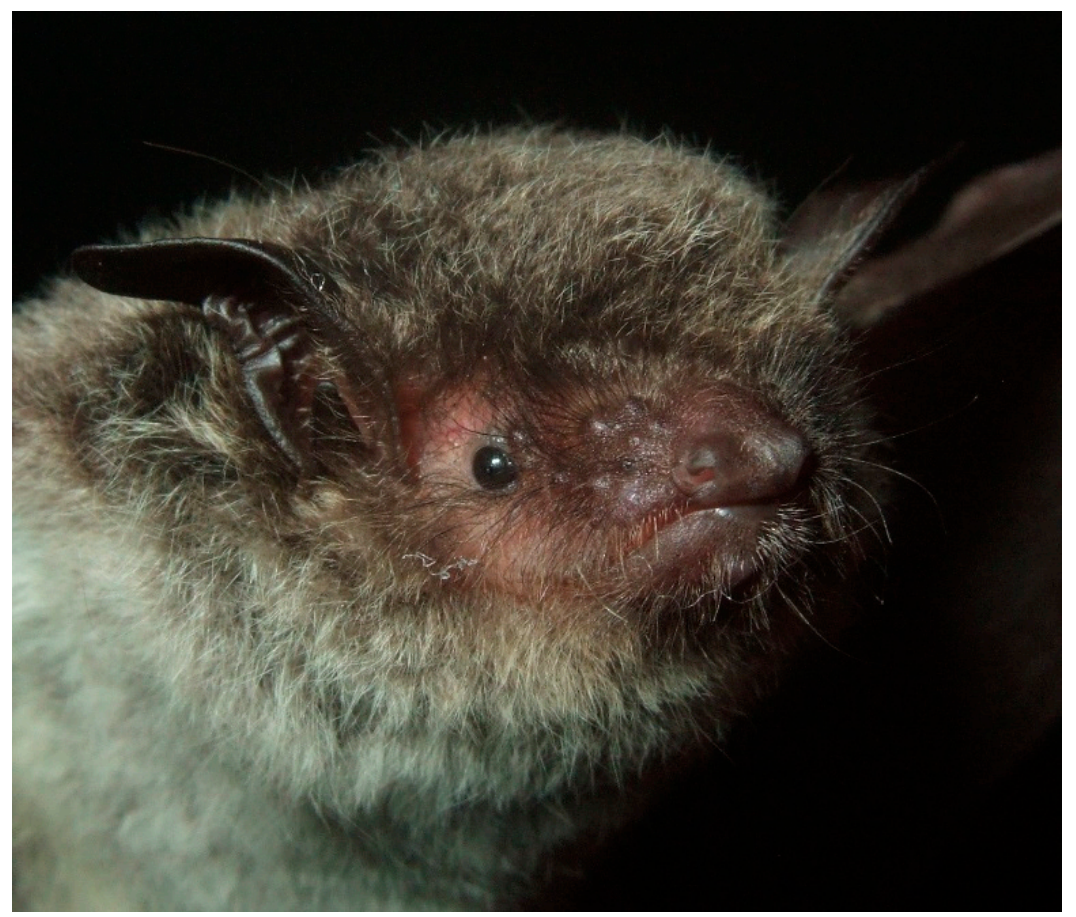

Figure 18. Myotis hasseltii, adult female Vu Dinh Thong. 


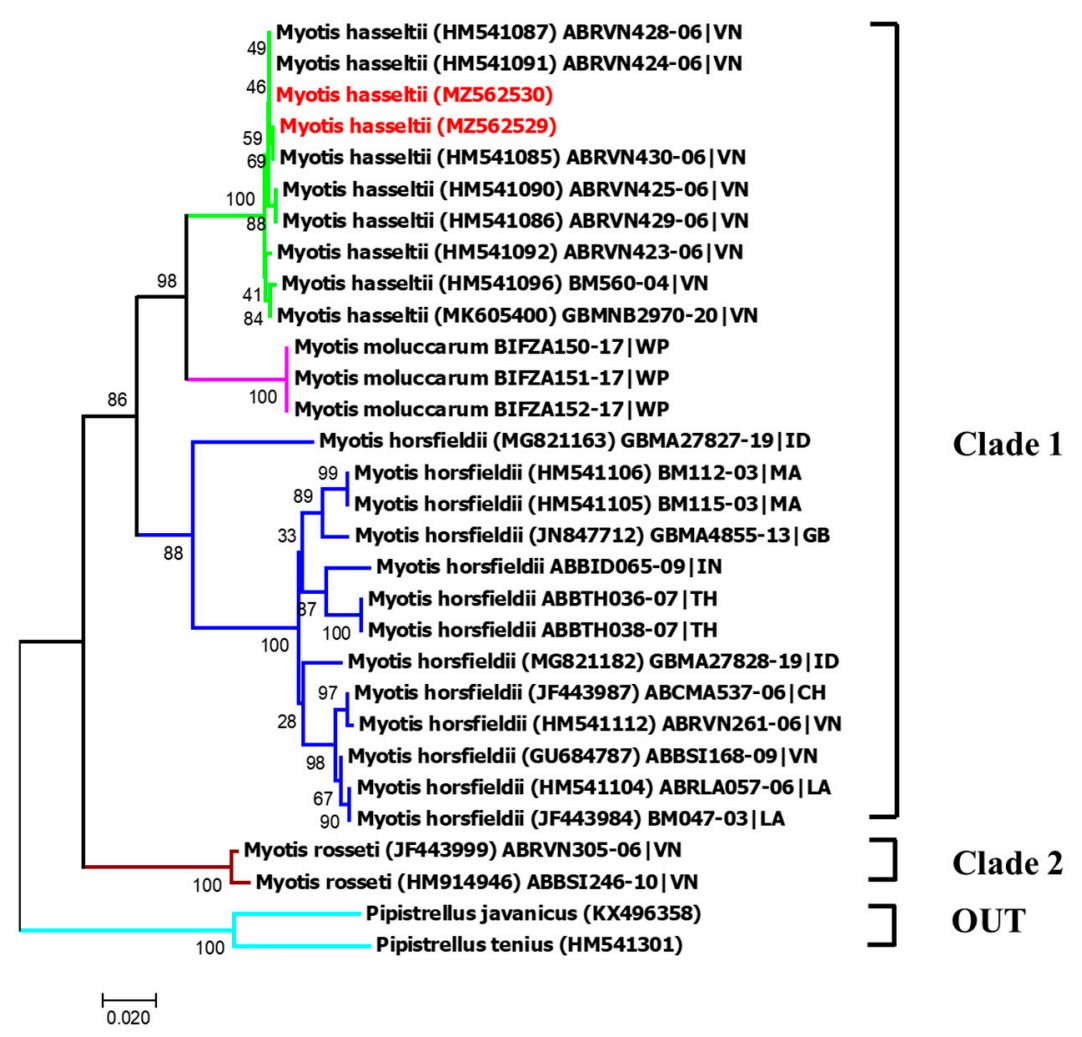

Figure 19. Molecular phylogenetic analysis of Myotis hasseltii samples collected in Cat Ba Island based on the aligned COI sequences. Two COI sequences generated in this study and 28 reference sequences obtained from the BOLD system were aligned by BioEdit software using ClustalW algorithm. The phylogenetic tree was built in MEGA v7.0. The bootstrap value with 1000 replicates is indicated next to each branch. The scale bar shows the number of nucleotide changes per site.

Phylogenetic analysis of M. hasseltii samples collected in Cat Ba (Figure 19) was created using 26 reference sequences of four Myotis species, Myotis hasseltii, Myotis moluccarum, Myotis horsfieldii, Myotis rosseti, and two sequences of Pipistrellus javanicus and Pipistrellus tenius as outgroup. The phylogenetic tree showed two major clades of the Myotis genus. Clade 1 comprises three species, Myotis hasseltii, Myotis moluccarum, Myotis horsfieldii while Clade 2 consists of a single species, Myotis rosseti. Clade 1 also shows three different groups including Myotis hasseltii, Myotis moluccarum, and Myotis horsfieldii (Figure 19). The two representative samples (GenBank accession numbers as MZ562529 and MZ562530) of $M$. hasseltii from Cat Ba Island are clustered in the M. hasseltii group with the high sequence similarity (>99\%). The COI sequence similarities between the M. hasseltii group and Myotis moluccarum, Myotis horsfieldii, and Myotis rosseti groups are $93 \%, 86 \%$, and $87 \%$, respectively. Therefore, the identification of $M$. hasseltii in this study is confirmed based on morphological and genetic data.

\subsubsection{Myotis pilosus}

Two males and two females were captured in mangroves and forests in Cat Ba Island and an unnamed island (Tables 1-3; Figure 20). Their morphological features are similar to previous descriptions [44,45]. Previous records of this species from Vietnam were regarded as Myotis ricketti [14]. However, Myotis ricketti is a synonym of this species [44,45]. It is distinguishable from other Myotis species in CBBR by body size and other morphological features $[18,27,44,45]$. This species is listed as "vulnerable" with a "very scattered" distribution in Vietnam [52]. However, it was commonly detected by acoustic recordings and observed in different habitats in the Cat Ba Island over the surveys. The iFM, tFM, and 
pulse duration values of four captured bats are in a range of $44.6-50.3 \mathrm{kHz}, 27.7-29.1 \mathrm{kHz}$, and $1.4-1.5 \mathrm{~ms}$, respectively.

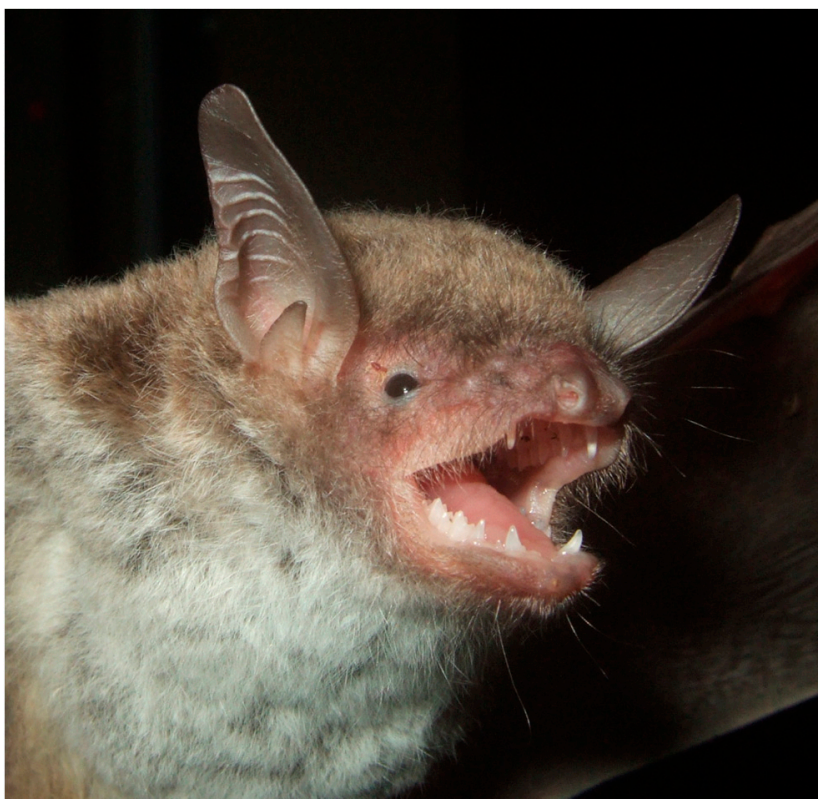

Figure 20. Myotis pilosus, adult male. Photograph by Vu Dinh Thong.

\subsubsection{Myotis alticraniatus}

One male and three females were captured in mangroves, forests, and plantations in Cat Ba Island (Tables 1-3; Figure 21). Morphological features of the four captured bats are identical to previous descriptions $[14,44,45]$. This species was commonly detected by acoustic recordings and observed in different habitats in Cat Ba Island. The iFM, tFM, and pulse duration values of four captured bats are in a range of $127.3-132.9 \mathrm{kHz}, 69.3-69.6 \mathrm{kHz}$, and $2.2-3.5 \mathrm{~ms}$, respectively.

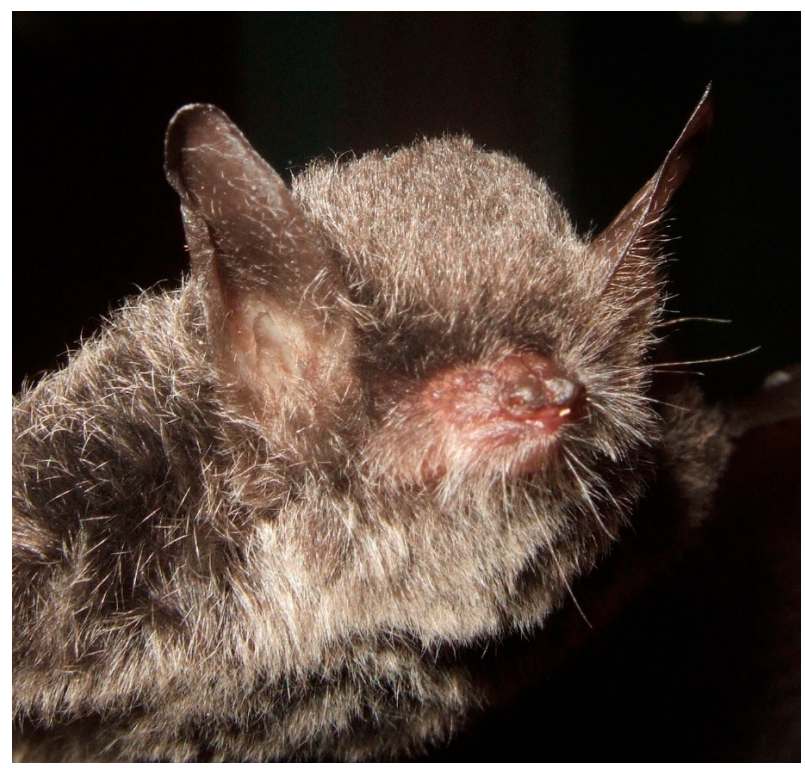

Figure 21. Myotis alticraniatus, adult male. Photograph by Vu Dinh Thong.

\subsubsection{Phoniscus jagorii}

One pregnant female was captured in a forest adjacent to a narrow stream near the Headquarters of Cat Ba National Park, Cat Ba Island (Tables 1 and 2; Figure 22). Before 
the present study, this species was rarely recorded from Vietnam. This is the first record of Phoniscus jagorii from CBBR. The pregnant female was released immediately after taking a photo and selected morphological measurements for identification. Its recorded calls were too faint to measure the sound parameters.

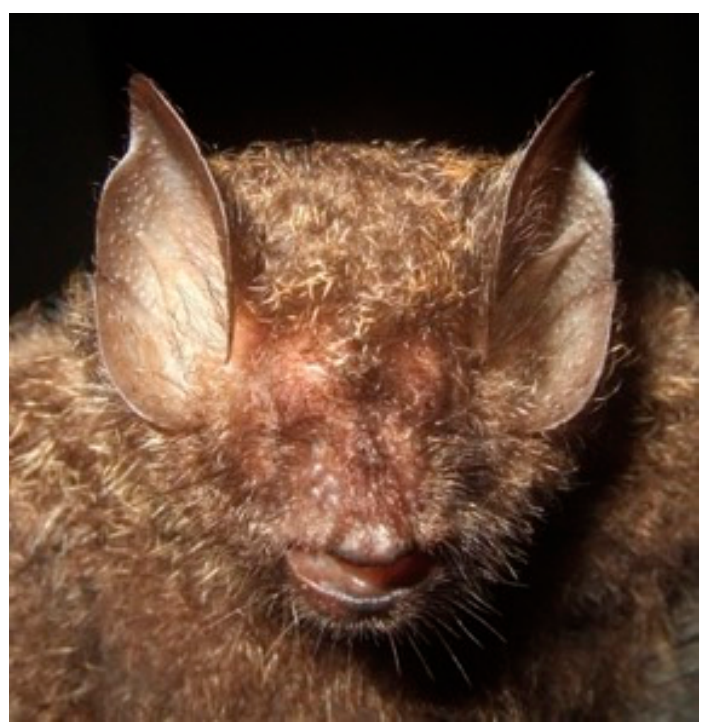

Figure 22. Phoniscus jagorii, adult female. Photograph by Vu Dinh Thong.

\subsubsection{Pipistrellus abramus}

One adult male was captured in a cave in the Cat Ba Island (Tables 1 and 2; Figure 23). Its morphological features are similar to previous descriptions $[14,44,45]$. This species is distinguishable from P. javanicus, a similar-sized Pipistrellus species recorded from Cat Ba Island and distinguishable using the shape of the canine and bacular structure [14]. This species is common in urban and agricultural habitats throughout the mainland and several islands of Vietnam. The iFM, tFM, and pulse duration values of one captured bat are in a range of $93.0-98.2 \mathrm{kHz}, 46.0-48.4 \mathrm{kHz}$, and $1.7-2.7 \mathrm{~ms}$, respectively.

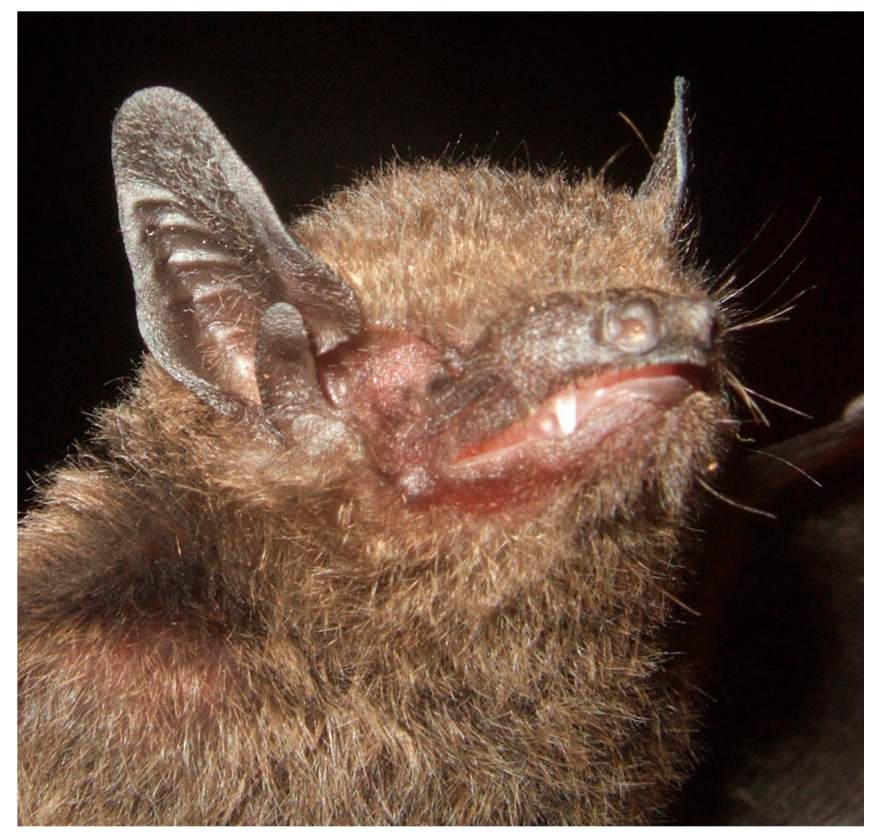

Figure 23. Pipistrellus abramus, adult male. Photograph by Vu Dinh Thong. 


\subsubsection{Tylonycteris fulvida}

One adult female was captured in a forest near the Headquarters of the Cat Ba National Park, Cat Ba Island (Table 1; Figure 24). Its morphological features are similar to previous descriptions [44]. This is the first record of a bamboo bat from CBBR. To date, three species of Tylonycteris are known from Vietnam: T. fulvida, T. malayana, and T. tonkinensis [44]. Of these, T. fulvida is distinguishable from the two remaining species by its body weight, and fluffier and more reddish fur [44]. With a weight of $3.3 \mathrm{~g}$ and fluffy fur, the captured bat is here identified as T. fulvida. The captured bat was kept as a specimen (IEBR-T.060819.1) deposited at the Institute of Ecology and Biological Resources (IEBR) for further studies of this species in the future. The $\mathrm{FMM}, \mathrm{tFM}$, and pulse duration values of one captured bat are $139.5 \mathrm{kHz}, 62.9 \mathrm{kHz}$, and $2.5 \mathrm{~ms}$, respectively.

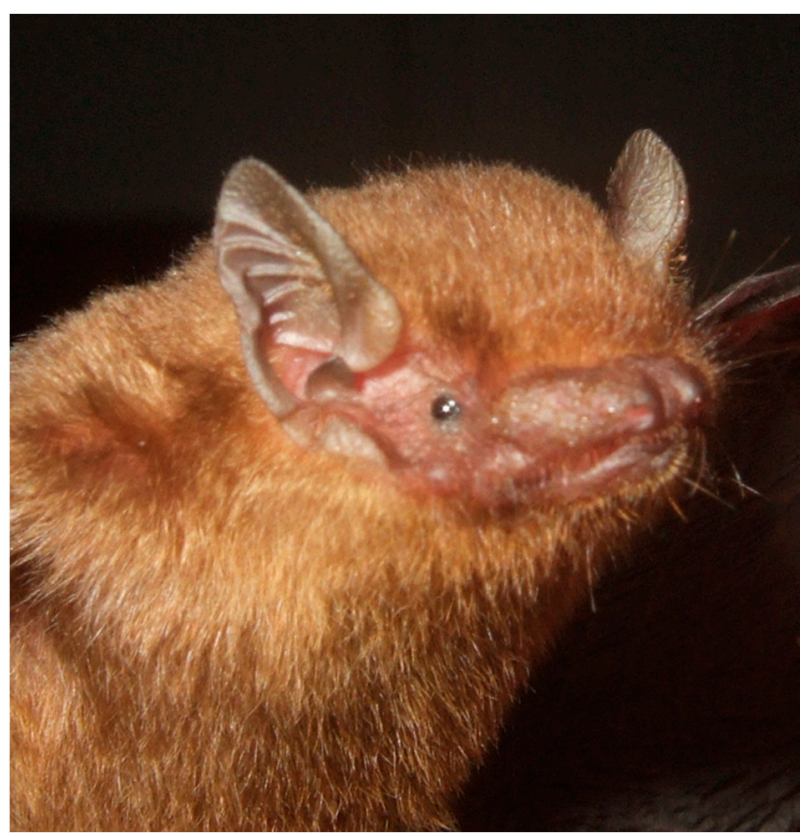

Figure 24. Tylonycteris fulvida, adult female. Photograph by Vu Dinh Thong.

\subsubsection{Miniopterus fuliginosus}

One female was recorded from a forest on an unnamed island (Tables 1-3). Its morphological features are similar to previous descriptions [44,45]. Previous records of this species were included in seven publications (Table 4). The records of "Miniopterus cf. fuliginosus" and "Miniopterus sp." in three publications must be regarded to this species (Table 4; Figure 25).

The forearm length of the individual captured in CBBR is larger than that of Miniopterus fuliginosus described in previous publications [14,44,45]. We examined the specimens, which were identified as "Miniopterus cf. fuliginosus" and "Miniopterus sp.", and recognized that their morphological features show the typical tragus shape of Miniopterus fuliginosus (Figure 25) in comparison with previous descriptions and illustrations [44,45]. This is known as a cave-dwelling species but has not been recorded at any cave in CBBR. This species likely inhabits at least an unsurveyed cave within the reserve. The iFM, $\mathrm{tFM}$, and pulse duration values of one captured bat are $89.7 \mathrm{kHz}, 42.6 \mathrm{kHz}$, and $3.8 \mathrm{~ms}$, respectively. 


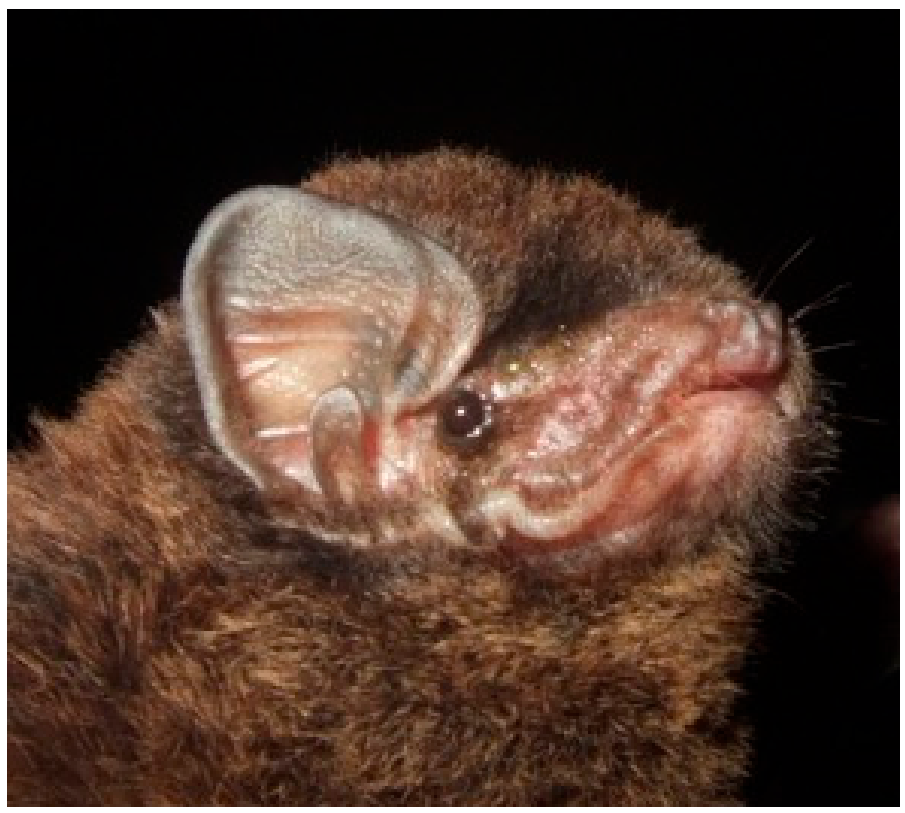

Figure 25. Miniopterus fuliginosus, adult female. Photograph by Vu Dinh Thong.

\subsection{Species Recorded by 2014}

\subsubsection{Cynopterus horsfieldii}

This species was included in two publications $[17,27]$. It was captured in a fruit tree garden near the Hoa Cuong Cave in Gia Luan Commune on Cat Ba Island [17]. To date, the peg-like cusps or ridges on the 3rd and 4th lower cheek teeth are the only morphological diagnosis to distinguish C. horsfieldii from other Cynopterus species [14,17,44,45]. However, genetic data do not support this morphological diagnosis. Results from molecular studies indicated that all specimens, with and without the peg-like cusps or ridges on the cheek teeth, are genetically similar to each other (Vu Dinh Thong, personal observation). Further investigation into materials of this species is required for a comprehensive taxonomic assessment in the future.

\subsubsection{Rousettus amplexicaudatus}

This species was included in four publications (Table 4). A female carrying a pup was captured on 26 June 2006 in a fruit tree garden near the headquarters of the Cat Ba National Park, Cat Ba Island. It was also observed in the plantation area near the headquarters $[9,23]$.

\subsubsection{Coelops frithii}

Furey included the first record of this species from the Cat Ba Island based on a single specimen that was captured at a site near the headquarters of the Cat Ba National Park [20]. This record was cited in five subsequent publications (Table 4). However, this species has not been recorded again in any of the twenty surveys conducted in Cat Ba National Park between 2006 and 2020.

\subsubsection{Hipposideros griffini}

This species was first included as an undescribed species, Hipposideros sp.nov, in Thong [23]. Its taxonomy and echolocation were subsequently described and confirmed in Thong [7] and Thong et al. [12], which were cited in four subsequent publications (Table 4). It was known as a cave-dwelling species in other areas in Vietnam [49,53], but has not been recorded in any caves in the Cat Ba Island. However, many caves in different islands in CBBR are still unsurveyed. This species may inhabit at least one of these caves. 


\subsubsection{Harpiocephalus harpia}

A record with reference to the specimen of this species was only included in Furey [20] which was cited in five subsequent publications (Table 4). This species has been rarely recorded throughout Vietnam.

\subsubsection{Murina harrisoni}

This species was first recorded as Murina tiensa from the Cat Ba Island by Thong et al. [8] which was cited in four subsequent publications (Table 4). However, Murina tiensa is a synonym of this species $[14,44,45]$.

\subsubsection{Pipistrellus javanicus}

The first record of this species was included in Canh et al. [21], which was cited in six subsequent publications (Table 4). However, the only specimen of this species from the Cat Ba Island was included in Furey [20]. This species is common in urban and other agricultural habitats in mainland Vietnam [14]. However, it has not been recorded from any urban or agricultural habitat in Cat Ba Island between 2006 and 2020.

\subsubsection{Pipistrellus tenuis}

Records of this species from Cat Ba National Park were included in seven publications (Table 4). In mainland Vietnam, this species is abundant in agricultural and urban habitats [14]. However, it was not recorded in any agricultural or urban habitat in CBBR during the recent surveys.

\subsection{Notes on Unconfirmed Historical Records}

Canh et al. listed two species, Scotophilus heathi and Scotophilus kuhlii, without reference to any specimen or information sources [21]. Subsequently, these two species were cited in at least four publications $[9,23,24,27]$. To date, the occurrence of these two species in CBBR is still under discussion. Therefore, we did not include these two species in the current bat species composition in CBBR.

\section{Conclusions}

Cat Ba Biosphere is home to at least 32 species belonging to 16 genera and six families. It has a high potential for bat diversity since the recorded number of bat species has increased continuously over the past decades while many islands and areas of the reserve remain unsurveyed. Mangrove is therefore a very important ecosystem for bat research and conservation.

Author Contributions: Conceptualization, V.D.T.; methodology, V.D.T., A.D. and H.-U.S.; validation, V.D.T., A.D., H.-U.S.; formal analysis, V.D.T., N.V.S. and N.T.T.H.; investigation, V.D.T., A.D. and H.U.S.; resources, V.D.T., A.D. and H.-U.S.; data curation, V.D.T., N.V.S., N.T.T.H., D.N.L., P.V.N., H.T.T., N.V.V., P.D.T., J.C.-C.H., N.T.L. and L.T.; writing-original draft preparation, V.D.T.; writing-review and editing, A.D., J.C.-C.H., M.-N.T., H.-U.S. All authors have read and agreed to the published version of the manuscript.

Funding: This research is funded by the Vietnam National Foundation for Science and Technology Development (NAFOSTED) under the grant number 106.05-2017.35.

Institutional Review Board Statement: The study was approved by the Institutional Review Board of the Institute of Ecology and Biological Resources (protocol code 751/STTNSV approved on 26 August 2015).

Data Availability Statement: Not applicable.

Acknowledgments: We thanks the Vietnam National Foundation for Science and Technology Development for support under the grant number 106.05-2017.35; the Asian Soundscape Monitoring Network funded by TIEN*Cooperation Center (Asi@Connect-17-100) and Academia Sinica; Paul A. Racey of the University of Exeter, UK; Paul J.J. Bates and David Harrison of the Harrison Institute, 
England; Neil M. Furey of the Fauna and Flora International, Cambodia Programme; Tigga Kingston of the Texas Tech University, USA; Marianne Carter, Robyn Dalzen, Stuart Paterson, Christina Imrich, Henry Rees, Leala Rosen, Kate Toiton and Sherilyn Bos of the Conservation Lead-ership Programme; Doctorates of the Institute of Ecology and Biological Resources, VAST, and Cat Ba National Park; Hoang Van Thap, Vu Hong Van and Nguyen Xuan Khu of Cat Ba National Park; Nguyen Tran Vy of the Institute of Tropical Biology, VAST for their various support.

Conflicts of Interest: The authors declare no conflict of interest.

\section{References}

1. Tuyen, L.T.; Tri, N.H. Cat Ba Archipelago Biosphere Reserve: Community participation and quality economy development. In Proceedings of the 7th Southeast Asian Biosphere Reserve Network Meeting, Puerto Princesa, Palawan, Phillippines, 24-26 October 2013.

2. Bourret, R. Sur quelques petits Mammifères du Tonkin et du Laos. In Comptes Rendus du Conseil de Recherches Scientifiques de L'indochine 2ème Semester; Université Indochinoise: Hanoi, Vietnam, 1942; pp. 27-30.

3. Bourret, R. Les Mammifères de la Collection du Laboratoire de Zoologie de l'École Supérieure des Sciences; Université Indochinoise: Hanoi, Vietnam, 1942; pp. 1-13.

4. Topál, G. Taxonomic status of Hipposideros larvatus alongensis Bourret, 1942 and the occurrence of H. turpis Bangs, 1901 in Vietnam (Mammalia, Chiroptera). Acta Zool. Hung. 1993, 39, 267-288.

5. Thong, V.D.; Tu, V.T.; Tien, P.D.; Chu, C.-W.; Senawi, J.; Bates, P.J.J.; Furey, N.M. Echolocation call frequency of Marshall's horseshoe bat Rhinolophus marshalli from Cat Ba National Park and its current status in Vietnam. In Proceedings of the 2nd National Scientific Conference on Ecology and Biological Resources, Hanoi, Vietnam, 26 October 2007; pp. $274-277$.

6. Thong, V.D.; Dietz, C.; Schnitzler, H.-U.; Denzinger, A.; Furey, N.M.; Borissenko, A.; Bates, P.J.J. First record of Hipposideros khaokhouayensis (Chiroptera: Hipposideridae) from Vietnam. HNUE J. Sci. 2008, 53, 138-143.

7. Thong, V.D. Systematics and Echolocation of Rhinolophoid Bats (Mammalia: Chiroptera) in Vietnam. Ph.D. Thesis, University of Tuebingen, Tuebingen, Germany, 26 March 2011.

8. Thong, V.D.; Dietz, C.; Denzinger, A.; Bates, P.J.J.; Furey, N.M.; Csorba, G.; Hoye, G.; Thuy, L.D.; Schnitzler, H.-U. Further records of Murina tiensa from Vietnam with first information on its echolocation calls. Hystrix Ital. J. Mammal. 2011, 22, 129-138.

9. Abramov, A.V.; Kruskop, S.V. The mammal fauna of Cat Ba Island, northern Vietnam. Russ. J. Theriol. 2012, 11, 57-72. [CrossRef]

10. Thong, V.D. New findings and an extensive description of Rhinolophus marshalli Thonglongya, 1973 in Vietnam. HNUE J. Sci. 2012, 57, 3-10.

11. Thong, V.D.; Puechmaille, S.J.; Denginger, A.; Bates, P.J.J.; Dietz, C.; Csorba, G.; Soisook, P.; Teeling, E.C.; Matsumura, S.; Furey, N.M.; et al. Systematics of the Hipposideros turpis complex and a description of a new subspecies from Vietnam. Mammal Rev. 2012, 42, 166-192. [CrossRef]

12. Thong, V.D.; Puechmaille, S.J.; Denzinger, A.; Dietz, C.; Csorba, G.; Bates, P.J.J.; Teeling, E.C.; Schnitzler, H.-U. A new species of Hipposideros (Chiroptera: Hipposideridae) from Vietnam. J. Mammal. 2012, 93, 1-11. [CrossRef]

13. Thong, V.D.; Dietz, C.; Denzinger, A.; Bates, P.J.J.; Puechmaille, S.J.; Callou, C.; Schnitzler, H.-U. Resolving a mammal mystery: The identity of Paracoelops megalotis (Chiroptera: Hipposideridae). Zootaxa 2012, 3505, 75-85. [CrossRef]

14. Kruskop, S.V. Bats of Vietnam: Checklist and an Identification Manual, 2nd ed.; KMK Ltd.: Moscow, Russia, 2013 ; pp. 1-300.

15. Thong, V.D. An updated list of leaf-nosed bats (Hipposideridae) from Vietnam and key features of Hipposideros alongensis. Tap Chi Sinh Hoc (Vietnam. J. Biol.) 2013, 35, 178-184. [CrossRef]

16. Thong, V.D. Acoustic identification and taxonomic remarks of horseshoe bats (Chiroptera: Rhinolophidae) in Cat Ba National Park, north-eastern Vietnam. In Proceedings of the First International VAST-BAS Conference, Ha Long City, Vietnam, 20-21 November 2014; pp. 323-328. [CrossRef]

17. Thong, V.D. Taxonomy and ecology of Cynopterus horsfieldii (Chiroptera: Pteropodidae) from Vietnam. In Proceedings of the First International VAST-BAS Conference, Ha Long City, Vietnam, 20-21 November 2014; pp. 329-334. [CrossRef]

18. Thong, V.D.; Dung, D.T.; Thanh, N.V. An overview of bat research in Cat Ba Biosphere Reserve with remarks on previous records. In Proceedings of the 2nd National Scientific Conference on Biological Research and Teaching in Vietnam, Da Nang, Vietnam, 20 May 2016; pp. 737-744.

19. Yuzefovich, A.P.; Artyushin, I.A.; Kruskop, S.V. Not the Cryptic Species: Diversity of Hipposideros gentilis (Chiroptera: Hipposideridae) in Indochina. Diversity 2021, 13, 218. [CrossRef]

20. Furey, N. Fauna. Cat Ba National Park: Biodiversity Survey 1999; Furey, N., Canh, L.X., Fanning, E., Eds.; Frontier Vietnam Environmental Research Report 20; Society for Environmental Exploration, UK and Institute of Ecology and Biological Resources: Hanoi, Vietnam, 2002; pp. 18-30.

21. Canh, L.X.; Sung, C.V.; Lee, S.D. Mammal resources of Cat Ba and surrounding areas in Vietnam. In Ecosystem and Biodiversity of Cat Ba National Park and Ha Long Bay, Vietnam; Annals of Nature Conservation, The Korean National Council for Conservation of Nature, Korea, and Institute of Ecology and Biological Resources: Hanoi, Vietnam, 1997; Volume 12, pp. 147-159.

22. Borissenko, A.V.; Kruskop, S.V. Bats of Vietnam and Adjacent Territories: An Identification Manual; Joint Russian-Vietnamese Science and Technological Tropical Centre, Moscow and Hanoi, Russia and Vietnam: Moscow, Russia, 2003; pp. 1-212. 
23. Thong, V.D. Bat Conservation at Cat Ba Biosphere Reserve, North-east Vietnam, Conservation Leadership Programme, 2008. Available online: www.conservationleadershipprogramme.org (accessed on 19 May 2021).

24. Thong, V.D.; Furey, N.M. The bat fauna of Cat Ba Biosphere. Tap Chi Sinh Hoc (Vietnam. J. Biol.) 2008, 30, 73-77.

25. Can, D.N.; Endo, H.; Son, N.T.; Oshida, T.; Canh, L.X.; Phuong, D.H.; Lunde, D.P.; Kawada, S.-I.; Hayashida, A.; Sasaki, M. Checklist of Wild Mammal Species of Vietnam; Kyoto University, Japan, and Institute of Ecology and Biological Resources, Vietnam, Shoukadoh Book Sellers: Kyoto, Japan, 2008; pp. 68-182.

26. Nga, C.T.T.; Tung, N.S. Biodiversity research and conservation in Cat Ba National Park with updated records from recent field surveys. J. Viet. Environ. 2018, 9, 285-290. [CrossRef]

27. Thong, V.D.; Southaphan, S.; Nha, P.V. The conservation status of bats (Mammalia: Chiroptera) in Cat Ba National Park, Northern Vietnam. HNUE J. Sci. 2020, 65, 92-98.

28. Alongi, D.M. Present state and future of the world's mangrove forests. Environ. Conserv. 2002, 29, 331-349. [CrossRef]

29. Donato, D.C.; Kauffman, J.B.; Murdiyarso, D.; Kurnianto, S.; Stidham, M.; Kanninen, M. Mangroves among the most carbon-rich forests in the tropics. Nat. Geosci. 2011, 4, 293-297. [CrossRef]

30. Giri, C.; Ochieng, E.; Tieszen, L.L.; Zhu, Z.; Shingh, A.; Loveland, T. Status and distribution of mangrove forests of the world using earth observation satellite data. Glob. Ecol. Biogeogr. 2011, 20, 154-159. [CrossRef]

31. Dat, P.T.; Yoshino, K.; Kaida, N. Monitoring Mangrove Forest changes in Cat Ba Biosphere Reserve using ALOS PALSAR Imagery and a GIS-based Support Vector Machine Algorithm. In Advances and Applications in Geospatial Technology and Earth Resources; Tien, B.D., Ed.; Springer International Publishing AG: Cham, Switzerland, 2018; pp. 103-118.

32. Sikes, R.S.; Gannon, W.L.; The Animal Care and Use Committee of the American Society of Mammalogists. Guidelines of the American Society of Mammalogists for the use of wild mammals in research. J. Mammal. 2011, 92, 235-253. [CrossRef]

33. Sikes, R.S.; The Animal Care and Use Committee of the American Society of Mammalogists. 2016 Guidelines of the American Society of Mammalogists for the use of wild mammals in research and education. J. Mammal. 2016, 97, 663-688. [CrossRef]

34. Francis, C.M. A comparison of mist nets and two types of harp traps for capturing bats. J. Mammal. 1989, 70, 865-870. [CrossRef]

35. Bates, P.J.J.; Harrison, D.L. Bats of the Indian Subcontinent; Harrison Zoological Museum: Sevenoaks, UK, 1997 ; pp. 1-258.

36. Csorba, G.; Ujhelyi, P.; Thomas, N. Horseshoe Bats of the World (Chiroptera: Rhinolophidae); Alana Books: Shropshire, UK, 2003; pp. $1-160$.

37. Racey, P.A. Reproductive assessment in bats. In Ecological and Behavioral Methods for the Study of Bats; Kunz, T.H., Parsons, S., Eds.; Johns Hopkins University Press: Baltimore, ML, USA, 2009; pp. 249-264.

38. Brunet-Rossinni, A.K.; Wilkinson, G.S. Methods for age estimation and the study of senescence in bats. In Ecological and Behavioral Methods for the Study of Bats; Kunz, T.H., Parsons, S., Eds.; Johns Hopkins University Press: Baltimore, ML, USA, 2009 ; pp. 315-325.

39. Ivanova, N.V.; Dewaard, J.R.; Hebert, P.D. An inexpensive, automation-friendly protocol for recovering high-quality DNA. Mol. Ecol. Notes 2006, 6, 998-1002. [CrossRef]

40. Hall, T.; BioEdit. A user-friendly biological sequence alignment editor and analysis program for Windows 95/98/NT. Nucleic Acids Symp. 1999, 41, 95-98.

41. Kumar, S.; Stecher, G.; Tamura, K.; MEGA7. Molecular evolutionary genetics analysis version 7.0 for bigger datasets. Mol. Biol. Evol. 2016, 33, 1870-1874. [CrossRef] [PubMed]

42. Saitou, N.; Nei, M. The neighbor-joining method: A new method for reconstructing phylogenetic trees. Mol. Biol. Evol. 1987, 4, 406-425. [PubMed]

43. Tamura, K.; Đuley, J.; Nei, M.; Kumar, S. MEGA4: Molecular evolutionary genetics analysis (MEGA) software version 4.0. Mol. Biol. Evol. 2007, 24, 1596-1599. [CrossRef]

44. Wilson, D.E.; Mittermerier, R.A.; Martinez-Vilalta, A.; Leslie, D.M.; Olive, M.; Elliott, A.; Velikov, I.; Mascarell, A.; Sogorb, L.; Marti, B.; et al. Handbook of the Mammals of the World; Lynx Edicions: Barcelona, Spain, 2019; pp. 1-1008.

45. Francis, C. A Guide to the Mammals of South-East Asia, 2nd ed.; Bloomsbury Publishing Plc: London, UK, 2019 ; pp. 1-416.

46. Thong, V.D. First record of bats (Mammalia: Chiroptera) from mangrove in Dam Nai area, Ninh Thuan province, Central Vietnam. Acad. J. Biol. 2021, 43, 135-139.

47. Thanh, H.T.; Son, N.T.; Duong, V.T.; Luong, N.T.; Loi, D.N.; Thong, V.D. New records and morphological assessments of long-nosed fruit bats (chiroptera: Pteropodidae: Macroglossus spp.) from Vietnam. Acad. J. Biol. 2019, 41, 117-124. [CrossRef]

48. Thong, V.D.; Nha, P.V.; Tien, P.D.; Luong, N.T.; Dang, N.X.; Nghia, N.X.; Southaphanh, S.; Thongphachanh, L.; Dung, D.T.; Khu, N.X.; et al. Horseshoe bat species recorded in the mangrove ecosystem of the Cat Ba National Park, Northern Vietnam. Acad. J. Biol. 2021, 43, 127-133.

49. Thong, V.D. New records of griffin's leaf-nosed bat (Hipposideros griffini Thong et al. 2012) from Vietnam. J. Biol. 2012, $34,323-327$. [CrossRef]

50. Douangboubpha, B. Hipposideros khaokhouayensis (Amended Version of 2019 Assessment). The IUCN Red List of Threatened Species 2020: E.T136819A166602959. Available online: https:/ / dx.doi.org/10.2305/IUCN.UK.2020-1.RLTS.T136819A166602959 .en (accessed on 10 July 2021).

51. Tu, V.T.; Arai, A.; Kikuchi, F.; Hang, C.T.; Tuan, T.A.; Csorba, G.; Gorfol, T. Rediscovery of Van Hasselt's mouse-eared bat Myotis hasseltii (Temmink, 1840) and its first genetic data from Hanoi, northern Vietnam. J. Threat. Taxa 2019, 11, 13915-13919. [CrossRef] 
52. Jiang, T.L.; Feng, J.; Csorba, G.; Bates, P. Myotis pilosus. The IUCN Red List of Threatened Species 2019: E.T14193A22062554. 2019. Available online: http:/ / dx.doi.org/10.2305/IUCN.UK.2019-3.RLTS.T14193A22062554.en (accessed on 10 July 2021).

53. Thong, V.D. New records of Hipposideros griffini from lava caves and the threats to its conservation in Vietnam. Acad. J. Biol. 2019, 41,31-36. [CrossRef] 